\title{
ANALYSIS AND NUMERICAL SOLUTION OF COUPLED VOLUME-SURFACE REACTION-DIFFUSION SYSTEMS WITH APPLICATION TO CELL BIOLOGY
}

\author{
H. EGGER, K. FELLNER, J.-F. PIETSCHMANN, B.Q. TANG
}

\begin{abstract}
We consider the numerical solution of coupled volume-surface reaction-diffusion systems having a detailed balance equilibrium. Based on the conservation of mass, an appropriate quadratic entropy functional is identified and an entropy-entropy dissipation inequality is proven. This allows us to show exponential convergence to equilibrium by the entropy method. We then investigate the discretization of the system by a finite element method and an implicit time stepping scheme including the domain approximation by polyhedral meshes. Mass conservation and exponential convergence to equilibrium are established on the discrete level by arguments similar to those on the continuous level and we obtain estimates of optimal order for the discretization error which hold uniformly in time. Some numerical tests are presented to illustrate these theoretical results. The analysis and the numerical approximation are discussed in detail for a simple model problem. The basic arguments however apply also in a more general context. This is demonstrated by investigation of a particular volume-surface reaction-diffusion system arising as a mathematical model for asymmetric stem cell division.
\end{abstract}

\section{INTRODUCTION}

Various physical phenomena in biology, material science, or chemical engineering are driven by reaction-diffusion processes in different compartments and by transfer between them. This may involve mass transfer between different domains but also with domain interfaces or boundaries. In cell-biology, for instance, many phenomena are based on reaction-diffusion processes of proteins within the cell-body and on the cell cortex [21,22]. Particular examples are systems modeling cell-biological signaling processes [16] or models for asymmetric stem cell division which describe the localization of so-called cell-fate determinants during mitosis [2, 20, 27].

As a simple model problem for such coupled volume-surface reaction-diffusion processes, we consider the system

$$
\begin{aligned}
\partial_{t} L-d_{L} \Delta L=0 & \text { on } \Omega, \\
\partial_{t} \ell-d_{\ell} \Delta_{\Gamma} \ell=\lambda L-\gamma \ell & \text { on } \Gamma, \\
d_{L} \partial_{\nu} L=-\lambda L+\gamma \ell & \text { on } \Gamma,
\end{aligned}
$$

which describes the diffusion of a substance with concentration $L$ in the volume and concentration $\ell$ on the surrounding surface coupled by mass transfer between these two compartments. Here $\Omega$ is the volume domain, $\Gamma=\partial \Omega$ is the surface, $\Delta_{\Gamma}$ denotes the Laplace-Beltrami operator, and the model parameters $d_{L}, d_{\ell}, \lambda, \gamma$ are assumed to be positive constants. The equations are supposed to hold for all $t>0$ and are complemented by appropriate initial conditions.

The system (1a)-(1c) may serve as a starting point for considering more realistic adsorption and desorption processes or as a reduced model for volume-surface reaction-diffusion processes

2010 Mathematics Subject Classification. 35K57, 65M60, 35B40.

Key words and phrases. reaction-diffusion systems, surface diffusion, finite element method, uniform error estimates, entropy method, exponential stability, detailed balance equilibrium, asymmetric stem cell division. 
arising in cell biology. But despite its simplicity, this model problem already features some interesting properties which will be of main interest for our further considerations:

(i) The system preserves non-negativity of solutions.

(ii) The total mass $M=\int_{\Omega} L d x+\int_{\Gamma} \ell d s$ is conserved for all time.

(iii) There exists a unique constant positive detailed balance equilibrium which can be parametrized explicitly by the total mass $M$ and the model parameters $\lambda$ and $\gamma$.

(iv) The solutions are uniformly bounded and converge exponentially fast towards the equilibrium state with respect to any reasonable Lebesgue or Sobolev norm.

The first goal of our paper will be to establish these properties, in particular (iii) and (iv), for the model problem (1a)-(1c). Based on this analysis, the second aim is then to investigate the systematic discretization of the system by finite elements and implicit time-stepping schemes. The guideline for the construction of the numerical approximation is to preserve and utilize the key features of the model as far as possible also for the analysis on the discrete level. In particular, we will establish the conservation of mass, prove the existence of a unique discrete equilibrium, and show the exponential convergence to equilibrium on the discrete level. We also comment briefly on the possibility for preserving the non-negativity of solutions.

Our arguments are closely related to the concepts of structure-preserving schemes, geometric integration, and compatible discretization that have been developed over the last decades. We refer the reader to the extensive survey [7] and the references therein. Several results concerning the preservation of entropic structures for discretization schemes have been obtained more recently, see e.g. $[3,4,6,18,19]$. This paper adds to this research by addressing the analysis and numerical solution of coupled volume-surface reaction-diffusion systems.

Apart from the entropy arguments, the numerical analysis is based on standard tools for the discretization of evolution problems [25, 26], on previous work concerning the analysis of domain approximations $[1,9,10,11]$, and on recent results from [13], who considered a somewhat simpler stationary volume-surface reaction-diffusion problem. The entropy estimates will allow us to establish order optimal convergence estimates with constants that are uniform in time.

The model problem (1a)-(1c) is simple enough to avoid complicated notation and to present our basic ideas in the most convenient way to the reader. To illustrate the applicability to more general problems, we consider in Section 7 the following system which models the evolution of four conformations of the key protein Lgl during the mitosis of Drosophila SOP precursor stem-cells $[2,15,20]$.

$$
\begin{aligned}
\partial_{t} L-d_{L} \Delta L=-\beta L+\alpha P & \text { on } \Omega, \\
\partial_{t} P-d_{P} \Delta P=\beta L-\alpha P, & \text { on } \Omega, \\
\partial_{t} \ell-d_{\ell} \Delta_{\Gamma} \ell=-d_{L} \partial_{n} L+\chi_{\Gamma_{2}}(-\sigma \ell+\kappa p) & \text { on } \Gamma, \\
\partial_{t} p-d_{p} \Delta_{\Gamma}=\sigma \ell-\kappa p-d_{P} \partial_{n} P & \text { on } \Gamma_{2} .
\end{aligned}
$$

Note that the last equation only holds on a part $\Gamma_{2} \subseteq \Gamma$ of the boundary. The mass transfer between the domain and the surface is described by

$$
\begin{aligned}
d_{L} \partial_{n} L=-\lambda L+\gamma \ell & \text { on } \Gamma, \\
d_{P} \partial_{n} P=\chi_{\Gamma_{2}}(-\eta P+\xi p) & \text { on } \Gamma, \\
d_{p} \partial_{n_{\Gamma}} p=0 & \text { on } \partial \Gamma_{2} .
\end{aligned}
$$

The diffusion and reaction parameters are positive constants, and the equations are assumed to hold for $t>0$ and are complemented by appropriate initial conditions. In the cell-biological context, $L$ and $\ell$ denote the concentrations of native $L g l$ within the cell cytoplasm and on the cell cortex, while $P$ and $p$ denote the corresponding phosphorylated Lgl conformations. 
Let us point out that like the model problem (1a)-(1c), also the system (2a)-(2g) involves fully reversible reactions and mass transfer processes; see Figure 1 for a schematic sketch.
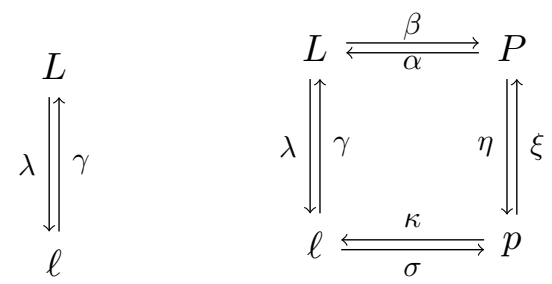

Figure 1. Mass transfer dynamics for model (1a)-(1c) (left) and the cellbiologically inspired model (2a)-(2g) (right). The top line represents the volume concentrations and the bottom line those on the surface. All reactions and mass transfer processes are reversible with constant reaction coefficients.

We will show that the key properties (i)-(iv) also hold for the system (2a)-(2g), i.e. solutions remain non-negative, the total mass is conserved, there exists a unique positive detailed balance equilibrium, and the concentrations converge exponentially fast to equilibrium. These assertions will be verified by minor modifications of the arguments already used for the analysis of the model problem (1a)-(1c). As a consequence, also the discretization strategy can be extended in a straight forward manner yielding approximations of optimal order and uniform in time.

The remainder of the manuscript is organized as follows: Section 2 is devoted to the analysis of problem (1a)-(1c) on the continuous level. In Section 3, we introduce the basic notation for the finite element discretization and recall some results about domain approximations. In Section 4, we analyze the semi-discretization in space. Section 5 is concerned with the time discretization by the implicit Euler method. The validity of the theoretical results is illustrated by some numerical tests in Section 6, and in Section 7 we show how to generalize our arguments to the model for asymmetric stem cell division mentioned above. The presentation closes with a short summary of our results and a discussion of open problems.

\section{Analysis of the Model PROBlem}

Let us start with introducing the relevant notation and basic assumptions and give a precise statement of our model problem.

2.1. Preliminaries. Let $\Omega \subset \mathbb{R}^{n}, n=2$ or 3 , be a bounded domain with sufficiently smooth boundary, say $\Gamma=\partial \Omega \in C^{3}$. We consider the volume-surface reaction-diffusion system

$$
\begin{aligned}
\partial_{t} L-d_{L} \Delta L=0, & x \in \Omega, t>0, \\
\partial_{t} \ell-d_{\ell} \Delta_{\Gamma} \ell=\lambda L-\gamma \ell, & x \in \Gamma, t>0,
\end{aligned}
$$

subject to the interface and initial conditions

$$
\begin{array}{cl}
d_{L} \partial_{n} L=\gamma \ell-\lambda L, & x \in \Gamma, t>0, \\
L(0)=L^{0}, \quad x \in \Omega, & \ell(0)=\ell^{0}, \quad x \in \Gamma .
\end{array}
$$

The diffusion coefficients $d_{L}, d_{\ell}$ and the reaction constants $\lambda, \gamma$ are assumed to be positive constants. We use standard notation for function spaces $[12]$ and we denote by $(\cdot, \cdot)_{\Omega}$ and $(\cdot, \cdot)_{\Gamma}$ the inner products and by $\|\cdot\|_{\Omega}$ and $\|\cdot\|_{\Gamma}$ the induced norms of $L^{2}(\Omega)$ and $L^{2}(\Gamma)$, respectively. 
2.2. Existence, uniqueness, and regularity. Let us briefly discuss the existence and uniqueness of weak solutions and their qualitative properties which we shall need later on.

Problem 1 (Weak formulation). Let $T>0$ be fixed. Find

$$
(L, \ell) \in C\left([0, T] ; L^{2}(\Omega) \times L^{2}(\Gamma)\right) \cap L^{2}\left(0, T ; H^{1}(\Omega) \times H^{1}(\Gamma)\right)
$$

such that $L(0)=L^{0}, \ell(0)=\ell^{0}$, and

$$
c\left(\partial_{t} L(t), \partial_{t} \ell(t) ; v, w\right)+a(L(t), \ell(t) ; v, w)=0
$$

for all $v \in H^{1}(\Omega)$ and $w \in H^{1}(\Gamma)$, and for a.e. $0<t<T$. The bilinear forms are given by

$$
a(L, \ell ; v, w):=d_{L}(\nabla L, \nabla v)_{\Omega}+d_{\ell}\left(\nabla_{\Gamma} \ell, \nabla_{\Gamma} w\right)_{\Gamma}+(\lambda L-\gamma \ell, v-w)_{\Gamma},
$$

and by $c(L, \ell ; v, w)=(L, v)_{\Omega}+(\ell, w)_{\Gamma}$, respectively.

This problem fits into the abstract framework of parabolic evolution equations as discussed, for instance, in $[8,12]$ and can be analyzed with standard arguments. A main ingredient is

Lemma 2 (Gårding inequality). For all $v \in H^{1}(\Omega)$ and $w \in H^{1}(\Gamma)$ there holds

$$
a(v, w ; v, w) \geq\|v\|_{H^{1}(\Omega}^{2}+\|w\|_{H^{1}(\Gamma)}^{2}-\eta\left(\|v\|_{\Omega}^{2}+\|w\|_{\Gamma}^{2}\right)
$$

with a constant $\eta>0$ depending only on the parameters $d_{L}, d_{\ell}, \gamma, \lambda$, and on the domain $\Omega$.

Proof. The result follows from the definition of $a(\cdot ; \cdot)$ and the Cauchy-Schwarz inequality.

Let us emphasize that the bilinear form $a(\cdot ; \cdot)$ is not elliptic which can easily be seen by choosing $v$ and $w$ as appropriate constants. Using the Gårding inequality and standard arguments for parabolic evolution problems, one readily obtains

Lemma 3 (Well-posedness of the weak formulation). For any pair of initial data $L^{0} \in L^{2}(\Omega)$ and $\ell^{0} \in L^{2}(\Gamma)$, the problem (3a)-(3d) has a unique weak solution $(L, \ell)$, and

$$
\sup _{t \in(0, T)}\left(\|L(t)\|_{\Omega}^{2}+\|\ell(t)\|_{\Gamma}^{2}\right)+\int_{0}^{T}\left(\|L(t)\|_{H^{1}(\Omega)}^{2}+\|\ell(t)\|_{H^{1}(\Gamma)}^{2}\right) d t \leq C_{T}\left(\left\|L^{0}\right\|_{\Omega}^{2}+\left\|\ell^{0}\right\|_{\Gamma}^{2}\right) .
$$

If the initial data are non-negative, then the solution remains non-negative for all time.

Proof. Existence and uniqueness of a global weak solution follow by standard results, see e.g., [8, Chapter XVIII], and positivity can be established by an iteration argument.

From the abstract analysis one obtains a-priori estimates with a constant $C_{T}$ that eventually increases with $T$. We will later show by entropy arguments that the bounds are actually uniform in time. A basic ingredient for our analysis will be the fact that the total mass is conserved during the evolution of our system.

Lemma 4 (Mass conservation). Let $(L, \ell)$ denote a weak solution of $(3 \mathrm{a})-(3 \mathrm{~d})$ and denote by

$$
M(t)=(L(t), 1)_{\Omega}+(\ell(t), 1)_{\Gamma}
$$

the total mass at time $t$. Then,

$$
M(t)=M(0)=: M^{0} \text { for all } t>0 .
$$

Proof. Testing the weak form (4) with $v \equiv 1$ and $w \equiv 1$, we get

$$
\frac{d}{d t} M(t)=\frac{d}{d t}(L(t), 1)_{\Omega}+\frac{d}{d t}(\ell(t), 1)_{\Gamma}=c\left(\partial_{t} L(t), \partial_{t} \ell(t) ; 1,1\right)=-a(L(t), \ell(t) ; 1,1)=0,
$$

and the result follows by integration with respect to time. 
2.3. Equilibrium system. A reaction-diffusion system like (3a)-(3c) can be assumed to eventually tend to equilibrium on the long run. For the problem under consideration, the equilibrium concentrations $L^{\infty}, \ell^{\infty}$ satisfy the stationary system

$$
\begin{aligned}
-d_{L} \Delta L^{\infty} & =0 & & \text { in } \Omega, \\
-d_{\ell} \Delta_{\Gamma} \ell^{\infty} & =\lambda L^{\infty}-\gamma \ell^{\infty} & & \text { on } \Gamma, \\
d_{L} \partial_{n} L^{\infty} & =\gamma \ell^{\infty}-\lambda L^{\infty} & & \text { on } \Gamma .
\end{aligned}
$$

Because of the mass conservation, we additionally know that

$$
\left(L^{\infty}, 1\right)_{\Omega}+\left(\ell^{\infty}, 1\right)_{\Gamma}=M^{0},
$$

where $M^{0}=\int_{\Omega} L^{0}+\int_{\Gamma} \ell^{0}$ is the total initial mass of the system. Note that this extra condition is already required to ensure the uniqueness of the equilibrium state. Using the above notation, the weak form of the equilibrium system is given by

Problem 5 (Equilibrium system). Find $L^{\infty} \in H^{1}(\Omega)$ and $\ell^{\infty} \in H^{1}(\Gamma)$ such that

$$
a\left(L^{\infty}, \ell^{\infty} ; v, w\right)=0,
$$

for all $v \in H^{1}(\Omega)$ and $w \in H^{1}(\Gamma)$ and such that the mass constraint $(6 \mathrm{~d})$ is satisfied.

For showing well-posedness of the equilibrium problem, we will utilize the following identity.

Lemma 6 (Inf-sup stability). For any $v \in H^{1}(\Omega)$ and $w \in H^{1}(\Gamma)$ there holds

$$
a(v, w ; \lambda v, \gamma w)=\lambda d_{L}\|\nabla v\|_{\Omega}^{2}+\gamma d_{\ell}\left\|\nabla_{\Gamma} w\right\|_{\Gamma}+\|\lambda v-\gamma w\|_{\Gamma}^{2} .
$$

As we will show next, the right hand side actually defines a norm on the space of functions with zero total mass. The following Poincaré-type inequality will also play a crucial role for the analysis of the time-dependent problem.

Lemma 7 (Poincaré-type inequality). There exists a constant $C_{P}>0$ depending only on the parameters $d_{L}, d_{\ell}, \lambda, \gamma$, and on the domain $\Omega$, such that

$$
\lambda\|L\|_{H^{1}(\Omega)}^{2}+\gamma\|\ell\|_{H^{1}(\Gamma)}^{2} \leq C_{P}\left(\lambda d_{L}\|\nabla L\|_{\Omega}^{2}+\gamma d_{\ell}\left\|\nabla_{\Gamma} \ell\right\|_{\Gamma}^{2}+\|\lambda L-\gamma \ell\|_{\Gamma}^{2}\right)
$$

for all $L \in H^{1}(\Omega)$ and $\ell \in H^{1}(\Gamma)$ satisfying the mass constraint $(L, 1)_{\Omega}+(\ell, 1)_{\Gamma}=0$.

Proof. The right hand side is zero if, and only if, $L$ and $\ell$ are constant and $L=\frac{\gamma}{\lambda} \ell$. Since the parameters $\gamma, \lambda$ are positive, the mass constraint yields $L=\ell=0$. Therefore, the term in parenthesis on the right hand side of (9) defines a norm on $H^{1}(\Omega) \times H^{1}(\Gamma)$. The uniform estimate is obtained by the lemma of equivalent norms; see e.g. [24, Ch. 11].

A combination of the previous two lemmas already yields the well-posedness of the equilibrium problem. Since the right hand side in (7) is zero, the solution can however even be obtained explicitly here. We will make use of the particular structure of the equilibrium later on.

Lemma 8 (Equilibrium). The system $(6 \mathrm{a})-(6 \mathrm{~d})$ has a unique weak solution $\left(L^{\infty}, \ell^{\infty}\right)$ given by

$$
L^{\infty}=\frac{\gamma M^{0}}{\gamma|\Omega|+\lambda|\Gamma|} \quad \text { and } \quad \ell^{\infty}=\frac{\lambda}{\gamma} L^{\infty} .
$$

Proof. One easily verifies that $\left(L^{\infty}, \ell^{\infty}\right)$ given by the formulas above is a solution of $(6 \mathrm{a})-(6 \mathrm{c})$ satisfying the mass constraint $(6 \mathrm{~d})$. Now assume that $\left(L^{*}, \ell^{*}\right)$ is any other weak solution to (6a) $-(6 \mathrm{~d})$. Then, the difference $\left(L^{\infty}-L^{*}, \ell^{\infty}-\ell^{*}\right)$ has zero total mass, and

$$
a\left(L^{\infty}-L^{*}, \ell^{\infty}-\ell^{*} ; v, w\right)=0
$$


for all $v \in H^{1}(\Omega)$ and $w \in H^{1}(\Gamma)$. By (8) we thus get

$$
\lambda d_{L}\left\|\nabla\left(L^{\infty}-L^{*}\right)\right\|_{\Omega}^{2}+\gamma d_{\ell}\left\|\nabla_{\Gamma}\left(\ell^{\infty}-\ell^{*}\right)\right\|_{\Gamma}^{2}+\left\|\lambda\left(L^{\infty}-L^{*}\right)-\gamma\left(\ell^{\infty}-\ell^{*}\right)\right\|_{\Gamma}^{2}=0,
$$

and Lemma 7 implies that $L^{\infty}=L^{*}$ and $\ell^{\infty}=\ell^{*}$. This shows the uniqueness.

2.4. Convergence to equilibrium. We will now show that the solution $(L(t), \ell(t))$ converges to the equilibrium $\left(L^{\infty}, \ell^{\infty}\right)$ exponentially fast by using the entropy method. For a given constant equilibrium state $\left(L^{\infty}, \ell^{\infty}\right)$, we define the quadratic relative entropy functional

$$
E(L, \ell)=\frac{1}{2}\left(\lambda\left\|L-L^{\infty}\right\|_{\Omega}^{2}+\gamma\left\|\ell-\ell^{\infty}\right\|_{\Gamma}^{2}\right),
$$

which is the square of a scaled $L^{2}$-distance to the equilibrium in the product space $L^{2}(\Omega) \times L^{2}(\Gamma)$.

Lemma 9 (Entropy dissipation). Let $(L, \ell)$ denote a weak solution of problem (3a)-(3d) with corresponding constant equilibrium state $\left(L^{\infty}, \ell^{\infty}\right)$. Then

$$
\begin{aligned}
\frac{d}{d t} E(L(t), \ell(t))=-d_{L} \lambda \| & \nabla\left(L(t)-L^{\infty}\right)\left\|_{\Omega}^{2}-d_{\ell} \gamma\right\| \nabla_{\Gamma}\left(\ell(t)-\ell^{\infty}\right) \|_{\Gamma}^{2} \\
- & \left\|\lambda\left(L(t)-L^{\infty}\right)-\gamma\left(\ell(t)-\ell^{\infty}\right)\right\|_{\Gamma}^{2}=:-D(L(t), \ell(t))
\end{aligned}
$$

for all $t>0$. The functional $D(\cdot)$ is called the entropy dissipation.

Let us emphasize that the entropy $E$ and the entropy dissipation $D$ depend on the equilibrium states $L^{\infty}$ and $\ell^{\infty}$. For ease of notation, we do not write this explicitly.

Proof. By definition of the entropy functional $E(\cdot)$ and elementary manipulations, we obtain

$$
\begin{aligned}
\frac{d}{d t} E(L(t), \ell(t)) & =\lambda\left(\partial_{t} L(t), L(t)-L^{\infty}\right)_{\Omega}+\gamma\left(\partial_{t} \ell(t), \ell(t)-\ell^{\infty}\right)_{\Gamma} \\
& =-a\left(L(t)-L^{\infty}, \ell(t)-\ell^{\infty} ; \lambda\left(L(t)-L^{\infty}\right), \gamma\left(\ell(t)-\ell^{\infty}\right)\right) \\
& =-d_{L} \lambda\left\|\nabla\left(L-L^{\infty}\right)\right\|_{\Omega}^{2}-d_{\ell} \gamma\left\|\nabla_{\Gamma}\left(\ell-\ell^{\infty}\right)\right\|_{\Gamma}^{2}-\left\|\lambda\left(L-L^{\infty}\right)-\gamma\left(\ell-\ell^{\infty}\right)\right\|_{\Gamma}^{2},
\end{aligned}
$$

which already yields the desired result.

From the Poincaré-type inequality established in Lemma 7, we may directly deduce

Lemma 10 (Entropy-entropy dissipation inequality). For all $L \in H^{1}(\Omega)$ and $\ell \in H^{1}(\Gamma)$ satisfying the mass constraint $(L, 1)_{\Omega}+(\ell, 1)_{\Gamma}=M^{0}$, there holds

$$
D(L, \ell) \geq c_{0} E(L, \ell) \quad \text { with } \quad c_{0}=2 / C_{P} .
$$

A combination of the previous estimates now immediately yields

Theorem 11 (Exponential stability). Let $(L, \ell)$ be the weak solution of the system (3a)-(3c) and $\left(L^{\infty}, \ell^{\infty}\right)$ denote the corresponding equilibrium. Then,

$$
\left\|L(t)-L^{\infty}\right\|_{\Omega}^{2}+\left\|\ell(t)-\ell^{\infty}\right\|_{\Gamma}^{2} \leq C e^{-c_{0} t}\left(\left\|L^{0}-L^{\infty}\right\|_{\Omega}^{2}+\left\|\ell^{0}-\ell^{\infty}\right\|_{\Gamma}^{2}\right)
$$

for all $t>0$ with constants $c_{0}, C>0$ depending only $d_{L}, d_{\ell}, \lambda, \gamma$, and on the domain $\Omega$.

Proof. As a direct consequence of Lemma 9 and 10, we get

$$
\frac{d}{d t} E(L(t), \ell(t)) \leq-c_{0} E(L(t), \ell(t)) \quad \text { for all } \quad t>0 .
$$

Therefore, the classical Gronwall inequality gives

$$
E(L(t), \ell(t)) \leq e^{-c_{0} t} E\left(L^{0}, \ell^{0}\right) .
$$

The desired result then follows from the fact that $\lambda, \gamma$ are positive constants and since the entropy $E(L, \ell)$ is equivalent to the square of the $L^{2}$-norm distance to equilibrium. 
Remark 12. The dependence of the constant $c_{0}$ on the parameters $\lambda, \gamma, d_{L}, d_{\ell}$, and on certain geometric constants can be made explicit, even for some nonlinear problems; see e.g. [14]. For linear problems, $c_{0}$ can also be determined from a generalized eigenvalue problem.

Remark 13. As a consequence of Theorem 11, we obtain uniform bounds for the solution in $L^{\infty}\left(0, \infty ; L^{2}(\Omega) \times L^{2}(\Gamma)\right)$. Since the problem (3a)-(3c) is linear and the coefficients are independent of time, one can obtain uniform bounds and even exponential decay also for $\left(\partial_{t}^{j} L, \partial_{t}^{j} \ell\right)$, $j \geq 0$ in $L^{p}\left(0, \infty ; H^{k}(\Omega) \times H^{k}(\Gamma)\right)$ provided that certain compatibility conditions hold; we refer to $[12$, Section 7$]$ for some basic results in this direction.

\section{BASIC NOTATION AND DOMAIN APPROXIMATIONS}

We now introduce the notation needed for the formulation of the finite element approximation of our problem and recall some basic results about the domain approximation by polyhedral meshes from $[9,13]$. For ease of presentation, we will only consider the two dimensional case here. All arguments however easily generalize to dimension three.

3.1. The mesh and domain mappings. Let $\Omega \subset \mathbb{R}^{2}$ be a bounded domain with smooth boundary $\Gamma \in C^{3}$. We approximate $\Omega$ by a polygonal domain $\Omega_{h}$ for which a conforming triangulation $T_{h}=\{T\}$ is available. As usual, we denote by $\rho_{T}$ and $h_{T}$ the incircle radius and the diameter of the triangle $T$, respectively, and we call $h=\max _{T} h_{T}$ the mesh size. We further denote by $E_{h}=\{e\}$ the partition of the domain boundary $\Gamma$ into edges $e$ inherited from the triangulation $T_{h}$. Throughout the subsequent sections, we make use of the following assumptions.

(A1) $T_{h}$ is $\gamma$-shape-regular, i.e., there exists a constant $\gamma>0$ such that

$$
\rho_{T} \leq h_{T} \leq \gamma \rho_{T}, \quad \text { for all } T \in T_{h} .
$$

(A2) There exists a diffeomorphism $G_{h}: \Omega_{h} \rightarrow \Omega$ such that the estimates

$$
\begin{array}{lll}
\left\|G_{h}-i d\right\|_{L^{\infty}(T)} \leq \beta h_{T}^{2}, & \left\|D G_{h}-I\right\|_{L^{\infty}(T)} \leq \beta h_{T}, & \left\|\operatorname{det}\left(D G_{h}\right)-1\right\|_{L^{\infty}(T)} \leq \beta h_{T}, \\
\left\|D G_{h}^{-1}\right\|_{L^{\infty}\left(G_{h}(T)\right)} \leq \beta, & \left\|D^{2} G_{h}\right\|_{L^{\infty}(T)} \leq \beta, & \left\|D^{2} G_{h}^{-1}\right\|_{L^{\infty}\left(G_{h}(T)\right)} \leq \beta
\end{array}
$$

for all $T \in T_{h}$ and $G_{h}(x)=x$ for all elements $T$ with $G_{h}(T) \cap \Gamma=\emptyset$.

(A3) The induced surface mapping $g_{h}: \Gamma_{h} \rightarrow \Gamma$ defined by $g_{h}=\left.G_{h}\right|_{\Gamma_{h}}$ in addition satisfies

$$
\left\|\operatorname{det}\left(D g_{h}\right)-1\right\|_{L^{\infty}(e)} \leq \beta h_{T}^{2}, \quad e \in E_{h}, e \subset \partial T \text {. }
$$

We will consider families of partitions $\left\{T_{h}\right\}_{h>0}$ later on, and the constants $\gamma, \beta$ are then assumed to be independent of $T_{h}$, in particular, of the mesh size. An explicit construction of appropriate domain mappings $G_{h}$ can be found in [13, Section 4.2]; see also [10, 11, 23].

3.2. Restriction to the discrete domain. To compare functions defined on $\Omega$ and $\Omega_{h}$, we associate to any $u: \Omega \rightarrow \mathbb{R}$ a function

$$
\widetilde{u}:=u \circ G_{h}
$$

defined on $\Omega_{h}$ called the restriction of $u$ to $\Omega_{h}$. Using the above properties of the domain mapping $G_{h}$, one easily verifies that

$$
c\|\widetilde{u}\|_{H^{k}(T)} \leq\|u\|_{H^{k}\left(G_{h}(T)\right)} \leq c^{-1}\|\widetilde{u}\|_{H^{k}(T)},
$$


for all $u \in H^{k}\left(G_{h}(T)\right)$ and $k \leq 2$ with a positive constant $c$ that only depends on $\beta$. In a similar manner, we define for any function $p: \Gamma \rightarrow \mathbb{R}$ defined on the boundary $\Gamma$ the restriction $\widetilde{p}$ to the boundary $\Gamma_{h}$ of the discrete domain by

$$
\widetilde{p}:=p \circ g_{h} .
$$

By the chain rule and application of the previous estimates, we readily obtain

$$
c\|\widetilde{p}\|_{H^{k}(e)} \leq\|p\|_{H^{k}\left(g_{h}(e)\right)} \leq c^{-1}\|\widetilde{p}\|_{H^{k}(e)}
$$

for all functions $p \in H^{k}(e)$ with $e \in E_{h}$ and integers $k \leq 2$.

\section{Semi-Discretization IN SPACE}

We now investigate the semi-discretization of problem $(3 \mathrm{a})-(3 \mathrm{c})$ in space. To this end, let

$$
V_{h}=\left\{v_{h} \in C\left(\Omega_{h}\right):\left.v_{h}\right|_{T} \in P_{1}(T) \quad \text { for all } T \in T_{h}\right\}
$$

be the space of continuous piecewise linear functions over $\Omega_{h}$, and denote by

$$
W_{h}=\left\{w_{h}: \Gamma_{h} \rightarrow \mathbb{R}: w_{h}=\left.v_{h}\right|_{\Gamma_{h}} \text { for some } v_{h} \in V_{h}\right\}
$$

the corresponding space of continuous piecewise linear functions on the surface $\Gamma_{h}$. Note that by construction $V_{h} \subset H^{1}\left(\Omega_{h}\right)$ and $W_{h} \subset H^{1}\left(\Gamma_{h}\right)$. Moreover, $W_{h}$ contains all traces of functions in $V_{h}$ which we will use later on.

4.1. Finite element discretization of the evolution problem. As approximation of the volume-surface reaction-diffusion system defined by (3a)-(3d), we consider

Problem 14 (Semi-discretization). Let $\left(L^{0}, \ell^{0}\right) \in L^{2}(\Omega)$ and set $\widetilde{L}^{0}=L^{0} \circ G_{h}$ and $\widetilde{\ell}^{0}=\ell^{0} \circ g_{h}$. Find $\left(L_{h}, \ell_{h}\right) \in H^{1}\left(0, T ; V_{h} \times W_{h}\right)$ such that

$$
\left(L_{h}(0), v_{h}\right)_{\Omega_{h}}=\left(\widetilde{L}^{0}, v_{h}\right)_{\Omega_{h}} \quad \text { and } \quad\left(\ell_{h}(0), w_{h}\right)_{\Gamma_{h}}=\left(\widetilde{\ell}^{0}, w_{h}\right)_{\Gamma_{h}},
$$

for all $v_{h} \in V_{h}, w_{h} \in W_{h}$ and such that

$$
c_{h}\left(\partial_{t} L_{h}(t), \partial_{t} \ell_{h}(t), v_{h}, w_{h}\right)+a_{h}\left(L_{h}, \ell_{h} ; v_{h}, w_{h}\right)=0
$$

holds for all $v_{h} \in V_{h}$ and $w_{h} \in W_{h}$, and for all $0<t \leq T$. The bilinear forms are given by

$$
a_{h}(L, \ell ; v, w)=d_{L}(\nabla L, \nabla v)_{\Omega_{h}}+d_{\ell}\left(\nabla_{\Gamma_{h}} \ell, \nabla_{\Gamma_{h}} w\right)_{\Gamma_{h}}+(\lambda L-\gamma \ell, v-w)_{\Gamma_{h}} .
$$

and $c_{h}(L, \ell ; v, w)=(L, v)_{\Omega_{h}}+(\ell, w)_{\Gamma_{h}}$, respectively.

Note that the variational principle (20) has the same structure as the weak form (4) of the continuous problem which allows us to utilize similar arguments as in Section 2 for the analysis of the discrete problem. By choice of a basis for the finite dimensional spaces $V_{h}$ and $W_{h}$, Problem 14 can be recast as a linear system of ordinary differential equations. Existence and uniqueness of a solution then follow immediately from the Picard-Lindelöf theorem.

Lemma 15. For any $L^{0} \in L^{2}(\Omega)$ and $\ell^{0} \in L^{2}(\Gamma)$, Problem 14 has a unique solution.

As a next step, let us note that the total mass is conserved also on the discrete level. Choosing $v_{h} \equiv 1$ and $w_{h} \equiv 1$ as test functions in (20), we obtain similar to the continuous level

Lemma 16 (Mass conservation). Let $\left(L_{h}, \ell_{h}\right)$ denote the solution of Problem 14. Then

$$
\left(L_{h}(t), 1\right)_{\Omega_{h}}+\left(\ell_{h}(t), 1\right)_{\Gamma_{h}}=\left(\widetilde{L}^{0}, 1\right)_{\Omega_{h}}+\left(\widetilde{\ell}^{0}, 1\right)_{\Gamma_{h}}=: M_{h}^{0},
$$

for all $t>0$, i.e., the total mass is conserved for all times also on the discrete level. 
Remark 17. With the usual mass lumping strategy, one could also preserve the non-negativity of the semi-discrete solution provided that the triangulation satisfies somewhat stronger conditions. We will not go into details here, but refer the reader to $[5,25]$ for details.

4.2. Geometric errors. As mentioned before, we can use the domain mappings $G_{h}: \Omega_{h} \rightarrow \Omega$ and $g_{h}: \Gamma_{h} \rightarrow \Gamma$ to define restrictions

$$
\widetilde{L}=L \circ G_{h} \quad \text { and } \quad \tilde{\ell}=\ell \circ g_{h},
$$

of the continuous solution onto the discrete domains which can then be compared with the discrete solution. Some properties of the restrictions have already been discussed in Section 3. For the numerical analysis, we now proceed with similar arguments as in $[10,13]$. However, we will work most of the time on the discrete domain $\Omega_{h}$ here instead of $\Omega$. To this end, let us also define the restriction of the bilinear form $a(\cdot ; \cdot)$ to the discrete domain $\Omega_{h}$ by

$$
\widetilde{a}(\widetilde{L}, \widetilde{\ell} ; \widetilde{v}, \widetilde{w}):=a(L, \ell ; v, w) \quad \text { for all } \quad(L, \ell),(v, w) \in H^{1}(\Omega) \times H^{1}(\Gamma) .
$$

Using the transformation formulas for integrals and derivatives, we can express $\widetilde{a}(\cdot ; \cdot)$ by

$$
\widetilde{a}(\widetilde{L}, \widetilde{\ell} ; \widetilde{v}, \widetilde{w})=d_{L}(A \nabla \widetilde{L}, \nabla \widetilde{v})_{\Omega_{h}}+d_{\ell}\left(B \nabla_{\Gamma_{h}} \widetilde{\ell}, \nabla_{\Gamma_{h}} \widetilde{w}\right)_{\Gamma_{h}}+(C(\lambda \widetilde{L}-\gamma \widetilde{\ell}), \widetilde{v}-\widetilde{w})_{\Gamma_{h}}
$$

with coefficient functions

$$
A=\left(D G_{h}^{\top} D G_{h}\right)^{-1} \operatorname{det}\left(D G_{h}\right), \quad B=\left(D g_{h}^{\top} D g_{h}\right)^{-1} \operatorname{det}\left(D g_{h}\right), \quad \text { and } \quad C=\operatorname{det}\left(D g_{h}\right) .
$$

The bilinear form $a_{h}(\cdot ; \cdot)$ used to define the finite element approximations can therefore be considered to be a non-conforming approximation of the bilinear form $\widetilde{a}(\cdot ; \cdot)$. In a similar manner, we define the restriction of the bilinear form $c(\cdot ; \cdot)$ by $\widetilde{c}(\widetilde{L}, \widetilde{\ell} ; \widetilde{v}, \widetilde{w}):=c(L, \ell ; v, w)$ which can also be expressed explicitly by

$$
\widetilde{c}(\widetilde{L}, \widetilde{\ell} ; \widetilde{v}, \widetilde{w}):=(K \widetilde{L}, \widetilde{v})_{\Omega_{h}}+(C \widetilde{\ell}, \widetilde{w})_{\Gamma_{h}}
$$

with $K=\operatorname{det}\left(D G_{h}\right)$ and $C=\operatorname{det}\left(D g_{h}\right)$ as before. The variational characterization (4) of the continuous solutions can then be written equivalently as

$$
\widetilde{c}\left(\partial_{t} \widetilde{L}(t), \partial_{t} \widetilde{\ell}(t) ; \widetilde{v}, \widetilde{w}\right)+\widetilde{a}(\widetilde{L}(t), \widetilde{\ell}(t) ; \widetilde{v}, \widetilde{w})=0
$$

for all $(\widetilde{v}, \widetilde{w}) \in H^{1}\left(\Omega_{h}\right) \times H^{1}\left(\Gamma_{h}\right)$, whereas the discrete variational problem (20) reads

$$
c_{h}\left(\partial_{t} L_{h}(t), \partial_{t} \ell_{h}(t) ; v_{h}, w_{h}\right)+a_{h}\left(L_{h}(t), \ell_{h}(t) ; v_{h}, w_{h}\right)=0
$$

for all $\left(v_{h}, w_{h}\right) \in V_{h} \times W_{h}$. Note that the semi-discretization (24) can thus be interpreted as a non-conforming approximation of problem (23) on the discrete domain. The differences between the bilinear forms $a_{h}(\cdot ; \cdot)$ and $\widetilde{a}(\cdot ; \cdot)$ as well as $c_{h}(\cdot ; \cdot)$ and $\widetilde{c}(\cdot ; \cdot)$ are only due to geometric errors that can be quantified explicitly with similar arguments as in [13, Lemma 6.2].

Lemma 18. For all $(L, \ell)$ and $(v, w) \in H^{1}(\Omega) \times H^{1}(\Gamma)$ there holds

$$
\left|a_{h}(\widetilde{L}, \widetilde{\ell} ; \widetilde{v}, \widetilde{w})-\widetilde{a}(\widetilde{L}, \widetilde{\ell} ; \widetilde{v}, \widetilde{w})\right| \leq C h\|(L, \ell)\|_{H^{1}(\Omega) \times H^{1}(\Gamma)}\|(\widetilde{v}, \widetilde{w})\|_{H^{1}\left(\Omega_{h}\right) \times H^{1}\left(\Gamma_{h}\right)} .
$$

The constant $C$ is independent of the mesh size $h$.

Proof. The estimates follow from [13, Lemma 6.2] with minor modifications in the proofs.

We will later in particular also need the following estimate for the error in the bilinear form $c$. 
Lemma 19. For all $(L, \ell)$ and $(v, w) \in L^{2}(\Omega) \times L^{2}(\Gamma)$ there holds

$$
\left|c_{h}(\widetilde{L}, \widetilde{\ell} ; \widetilde{v}, \widetilde{w})-\widetilde{c}(\widetilde{L}, \widetilde{\ell} ; \widetilde{v}, \widetilde{w})\right| \leq C h\|(L, \ell)\|_{L^{2}(\Omega) \times L^{2}(\Gamma)}\|(\widetilde{v}, \widetilde{w})\|_{L^{2}\left(\Omega_{h}\right) \times L^{2}\left(\Gamma_{h}\right)} .
$$

If in addition $L, v \in H^{1}(\Omega)$ and $\ell, w \in H^{1}(\Gamma)$, then

$$
\left|c_{h}(\widetilde{L}, \widetilde{\ell} ; \widetilde{v}, \widetilde{w})-\widetilde{c}(\widetilde{L}, \widetilde{\ell} ; \widetilde{v}, \widetilde{w})\right| \leq C h^{2}\|(L, \ell)\|_{H^{1}(\Omega) \times H^{1}(\Gamma)}\|(\widetilde{v}, \widetilde{w})\|_{H^{1}\left(\Omega_{h}\right) \times H^{1}\left(\Gamma_{h}\right)} .
$$

The constant $C$ in these estimates is again independent of the mesh size $h$.

As will become clear from the proof, the regularity requirements could be somewhat relaxed.

Proof. By definition of the bilinear forms, we have

$$
c_{h}(\widetilde{L}, \widetilde{\ell} ; \widetilde{v}, \widetilde{w})-\widetilde{c}(\widetilde{L}, \widetilde{\ell} ; \widetilde{v}, \widetilde{w})=\int_{B_{h}} \widetilde{L} \widetilde{v}\left(1-\operatorname{det}\left(D G_{h}\right)\right) d x+\int_{\Gamma_{h}} \widetilde{\ell} \widetilde{w}\left(1-\operatorname{det}\left(D g_{h}\right)\right) d s .
$$

with $B_{h}=\bigcup_{T \cap \Gamma_{h} \neq \emptyset} T$. Here we used that $G_{h}=i d$ for element not adjacent to the boundary due to assumption (A2). Using the bounds for the determinants in (A2) and (A3), we get

$$
c_{h}(\widetilde{L}, \widetilde{\ell} ; \widetilde{v}, \widetilde{w})-\widetilde{c}(\widetilde{L}, \widetilde{\ell} ; \widetilde{v}, \widetilde{w}) \leq C\left(h\|\widetilde{L}\|_{L^{2}\left(B_{h}\right)}\|\widetilde{v}\|_{L^{2}\left(B_{h}\right)}+h^{2}\|\widetilde{\ell}\|_{L^{2}\left(\Gamma_{h}\right)}\|\widetilde{w}\|_{L^{2}(\Gamma)}\right) .
$$

This already implies the first bound. A localized Poincaré inequality similar to [13, Lemma 4.10] and application of the trace theorem yields

$$
\|\widetilde{L}\|_{L^{2}\left(B_{h}\right)} \leq C\left(h^{1 / 2}\|\widetilde{L}\|_{L^{2}\left(\Gamma_{h}\right)}+h\|\nabla \widetilde{L}\|_{L^{2}\left(B_{h}\right)}\right) \leq C h^{1 / 2}\|\widetilde{L}\|_{H^{1}\left(\Omega_{h}\right)} .
$$

Using this and (17) to bound the first term in (25) yields the second assertion.

4.3. The discretization of the equilibrium problem. For the approximation of the equilibrium system (6a)-(6d), we consider the following discrete variational problem.

Problem 20 (Discrete equilibrium problem). Find $L_{h}^{\infty} \in V_{h}$ and $\ell_{h}^{\infty} \in W_{h}$ satisfying the mass constraint $\left(L_{h}^{\infty}, 1\right)_{\Omega_{h}}+\left(\ell_{h}^{\infty}, 1\right)_{\Gamma_{h}}=M_{h}^{0}$ and such that for all $\left(v_{h}, w_{h}\right) \in V_{h} \times W_{h}$ there holds

$$
a_{h}\left(L_{h}^{\infty}, \ell_{h}^{\infty} ; v_{h}, w_{h}\right)=0 .
$$

In order to ensure the well-posedness of this problem, it suffices to show uniqueness, which readily follows from the following two auxiliary results.

Lemma 21 (Discrete inf-sup stability). For any $v \in H^{1}\left(\Omega_{h}\right)$ and $w \in H^{1}\left(\Gamma_{h}\right)$ there holds

$$
a_{h}(v, w ; \lambda v, \gamma w)=\lambda d_{L}\|\nabla v\|_{\Omega_{h}}^{2}+\gamma d_{\ell}\left\|\nabla_{\Gamma_{h}} w\right\|_{\Gamma_{h}}^{2}+\|\lambda v-\gamma w\|_{\Gamma_{h}}^{2} .
$$

The right hand side of (27) defines a norm on the subspace of functions with zero total mass which follows by a Poincaré-type inequality. It will be important later on that the equivalence constant can be chosen to be independent of the mesh.

Lemma 22. For any $v \in H^{1}\left(\Omega_{h}\right)$ and $w \in H^{1}\left(\Gamma_{h}\right)$ with $(v, 1)_{\Omega_{h}}+(w, 1)_{\Gamma_{h}}=0$, there holds

$$
\lambda\|L\|_{H^{1}\left(\Omega_{h}\right)}^{2}+\gamma\|\ell\|_{H^{1}\left(\Gamma_{h}\right)}^{2} \leq C_{P}\left(\lambda d_{L}\|\nabla L\|_{\Omega_{h}}^{2}+\gamma d_{\ell}\left\|\nabla_{\Gamma} \ell\right\|_{\Gamma_{h}}^{2}+\|\lambda L-\gamma \ell\|_{\Gamma_{h}}^{2}\right)
$$

with a constant $C_{P}$ that only depends on the parameters $d_{L}, d_{\ell}, \lambda$, and $\gamma$, on the domain $\Omega$, and the constants $\beta, \gamma$ in assumptions (A1)-(A3) but is otherwise independent of the mesh $T_{h}$.

Proof. By transformation with the domain mapping $G_{h}$, all integrals in the definition of the norm can be cast into integrals over $\Omega$ and $\Gamma$, respectively. The assertion then follows from the Poincaré-type inequality stated in Lemma 7, the estimates for the geometric errors given in the previous section, and the norm equivalence estimates (17) and (19).

Since the constant $C_{P}$ can be chosen independently of the mesh size, we deliberately use the same symbol as on the continuous level here. By similar arguments as in Lemma 8, we obtain 
Lemma 23 (Discrete equilibrium). Problem 20 has a unique solution given by

$$
L_{h}^{\infty}=\frac{\gamma M_{h}^{0}}{\gamma\left|\Omega_{h}\right|+\lambda\left|\Gamma_{h}\right|} \quad \text { and } \quad \ell_{h}^{\infty}=\frac{\lambda}{\gamma} L_{h}^{\infty} .
$$

The error analysis for the equilibrium problem could be obtained by a careful extension of the results in [13]. Since the equilibrium is constant on the continuous and on the discrete level, we can however give also a direct proof of the discretization error estimate here.

Lemma 24. Let $\left(L^{\infty}, \ell^{\infty}\right)$ and $\left(L_{h}^{\infty}, \ell_{h}^{\infty}\right)$ be the solutions of $(7)$ and $(26)$, respectively Then

$$
\left|L^{\infty}-L_{h}^{\infty}\right|+\left|\ell^{\infty}-\ell_{h}^{\infty}\right| \leq C h^{2} .
$$

with a constant $C$ that is independent of the mesh size.

Proof. Using the explicit forms of $L^{\infty}$ and $L_{h}^{\infty}$ in (10) and (29) we can write

$$
\begin{aligned}
\left|L^{\infty}-L_{h}^{\infty}\right| & =\left|\frac{\gamma M^{0}}{\lambda|\Omega|+\gamma|\Gamma|}-\frac{\gamma M_{h}^{0}}{\lambda\left|\Omega_{h}\right|+\gamma\left|\Gamma_{h}\right|}\right| \\
& \leq c\left(\lambda|\Omega|\left|M^{0}-M_{h}^{0}\right|+\gamma M^{0}|| \Omega|-| \Omega_{h}||\right)
\end{aligned}
$$

The result for the volume term then follows from the fact that the solutions are constant and from the properties of $G_{h}$ stated in assumption (A2). The estimates for the boundary component follow in a similar manner.

4.4. Convergence to the discrete equilibrium. Following the basic steps of the analysis on the continuous level, we next define the discrete entropy functional

$$
E_{h}\left(L_{h}, \ell_{h}\right)=\frac{1}{2}\left(\lambda\left\|L_{h}-L_{h}^{\infty}\right\|_{\Omega_{h}}^{2}+\gamma\left\|\ell_{h}-\ell_{h}^{\infty}\right\|_{\Gamma_{h}}^{2}\right) .
$$

With the same arguments as in the proof of Lemma 9, we obtain

Lemma 25 (Discrete entropy dissipation). Let $\left(L_{h}, \ell_{h}\right)$ denote the solution of discrete evolution Problem 14 and $\left(L_{h}^{\infty}, \ell_{h}^{\infty}\right)$ be the corresponding discrete equilibrium. Then,

$$
\begin{aligned}
\frac{d}{d t} E_{h}\left(L_{h}(t), \ell_{h}(t)\right)=-d_{L} \lambda \| & \nabla\left(L_{h}(t)-L_{h}^{\infty}\right)\left\|_{\Omega_{h}}^{2}-d_{\ell} \gamma\right\| \nabla_{\Gamma}\left(\ell_{h}(t)-\ell_{h}^{\infty}\right) \|_{\Gamma_{h}}^{2} \\
- & \left\|\lambda\left(L_{h}(t)-L_{h}^{\infty}\right)-\gamma\left(\ell_{h}(t)-\ell_{h}^{\infty}\right)\right\|_{\Gamma_{h}}^{2}=:-D_{h}\left(L_{h}(t), \ell_{h}(t)\right) .
\end{aligned}
$$

By the discrete Poincaré-type inequality stated in Lemma 22 we further get

Lemma 26 (Discrete entropy-entropy dissipation inequality). For any $v \in H^{1}\left(\Omega_{h}\right)$ and every $w \in H^{1}\left(\Gamma_{h}\right)$ satisfying $(v, 1)_{\Omega_{h}}+(w, 1)_{\Gamma_{h}}=M_{h}^{0}$, there holds

$$
D_{h}(v, w) \geq c_{0} E_{h}(v, w) \quad \text { with } \quad c_{0}=2 / C_{P} .
$$

Note that $c_{0}$ can be chosen independently of $h$. Using the previous estimates, a Gronwall inequality, and the fact that the entropy is a scaled $L^{2}$-norm distance, we finally obtain

Theorem 27 (Discrete exponential stability). Let $\left(L_{h}, \ell_{h}\right)$ denote the solution to Problem 14 and $\left(L_{h}^{\infty}, \ell_{h}^{\infty}\right)$ be defined as in Lemma 23. Then

$$
\left\|L_{h}(t)-L_{h}^{\infty}\right\|_{\Omega_{h}}^{2}+\left\|\ell_{h}(t)-\ell_{h}^{\infty}\right\|_{\Gamma_{h}}^{2} \leq C e^{-c_{0} t}\left(\left\|\widetilde{L}^{0}-L_{h}^{\infty}\right\|_{\Omega_{h}}^{2}+\left\|\widetilde{\ell}^{0}-\ell_{h}^{\infty}\right\|_{\Gamma_{h}}^{2}\right),
$$

with constants $C>0$ and $c_{0}>0$ that are independent of $t$ and $h$.

Let us emphasize that up to perturbations which vanish with $h \rightarrow 0$, the constants $C$ and $c_{0}$ can be chosen the same as on the continuous level. The decay to equilibrium on the discrete level therefore occurs uniformly with respect o the mesh size and at the same rate as on the continuous level. This is also what we observe in our numerical tests; see Section 6 for details. 
4.5. Error estimates for the semi-discretization. The error analysis for the Galerkin approximation now proceeds with standard arguments $[25,26]$ but carefully taking into account the additional geometric errors. A key step is the definition of an appropriate operator $R_{h}: H^{1}(\Omega) \times H^{1}(\Gamma) \rightarrow V_{h} \times W_{h}$ that allows one to approximate the continuous solution $(L, \ell)$ by a function in the discretization space $V_{h} \times W_{h}$. For our purpose, we define $R_{h}$ by

$$
a_{h}\left(R_{h}(L, \ell) ; v_{h}, w_{h}\right)+\eta c_{h}\left(R_{h}(L, \ell) ; v_{h}, w_{h}\right)=\widetilde{a}\left(\widetilde{L}, \widetilde{\ell} ; v_{h}, w_{h}\right)+\eta c_{h}\left(\widetilde{L}, \widetilde{\ell} ; v_{h}, w_{h}\right)
$$

for all $\left(v_{h}, w_{h}\right) \in V_{h} \times W_{h}$. Note that the bilinear form $c_{h}$ appears on both sides in this definition. Following standard convention, we call $R_{h}$ elliptic projection, although it is not a projection in the strict sense. The basic properties of $R_{h}$ needed later on can be summarized as follows.

Lemma 28 (Elliptic projection). Let $\eta>0$ be chosen large enough. Then the elliptic projection $R_{h}: H^{1}(\Omega) \times H^{1}(\Gamma) \rightarrow V_{h} \times W_{h}$ is a well-defined bounded linear operator and the estimates

$$
\left\|(\widetilde{L}, \widetilde{\ell})-R_{h}(L, \ell)\right\|_{H^{1}\left(\Omega_{h}\right) \times H^{1}\left(\Gamma_{h}\right)} \leq C h\|(L, \ell)\|_{H^{2}(\Omega) \times H^{2}(\Gamma)}
$$

as well as

$$
\left\|(\widetilde{L}, \widetilde{\ell})-R_{h}(L, \ell)\right\|_{L^{2}\left(\Omega_{h}\right) \times L^{2}\left(\Gamma_{h}\right)} \leq C h^{2}\|(L, \ell)\|_{H^{2}(\Omega) \times H^{2}(\Gamma)}
$$

hold for all $L \in H^{2}(\Omega)$ and $\ell \in H^{2}(\Gamma)$ with a constant $C$ independent of the mesh size. Moreover,

$$
c_{h}\left(R_{h}(L, \ell) ; 1,1\right)=c_{h}(\widetilde{L}, \widetilde{\ell} ; 1,1) .
$$

This implies that the elliptic projection $R_{h}$ is mass preserving in a generalized sense.

Proof. By Lemma 2, the bilinear forms on both sides of (34) are elliptic for $\eta$ large enough. The elliptic projection therefore is a finite element approximation of an elliptic volume-surface reaction-diffusion problem. The error estimates then follow from the results in [13] with minor modification of the proofs. The conservation of mass is a direct consequence of the special construction of the elliptic projection and the fact that $a_{h}(\cdot, \cdot ; 1,1)=0$ and $\widetilde{a}(\cdot, \cdot ; 1,1)=0$.

We are now in the position to state and prove our first main result.

Theorem 29 (Error estimate for the semi-discretization). Let (A1)-(A3) hold and assume that the solution of problem (3a)-(3d) is sufficiently smooth. Then for all $t>0$ we have

$$
\left\|\widetilde{L}(t)-L_{h}(t)\right\|_{\Omega_{h}}+\left\|\widetilde{\ell}(t)-\ell_{h}(t)\right\|_{\Gamma_{h}} \leq C h^{2}
$$

with a constant $C$ that is independent of $t$ and $h$.

Proof. Set $U=(L, \ell), \widetilde{U}=(\widetilde{L}, \widetilde{\ell}), U_{h}=\left(L_{h}, \ell_{h}\right)$, and $R_{h} U=\left(\widetilde{L}_{R}, \widetilde{\ell}_{R}\right)$. In the usual manner, we decompose the error into

$$
U_{h}(t)-\widetilde{U}(t)=\left[\widetilde{U}(t)-R_{h} U(t)\right]+\left[U_{h}(t)-R_{h} U(t)\right]=: \widetilde{\rho}(t)+\theta_{h}(t) .
$$

By application of Lemma 28, we already obtain the desired estimate for the first component

$$
\|\widetilde{\rho}(t)\|_{L^{2}\left(\Omega_{h}\right) \times L^{2}\left(\Gamma_{h}\right)} \leq C h^{2}\|\widetilde{U}(t)\|_{H^{2}\left(\Omega_{h}\right) \times H^{2}\left(\Gamma_{h}\right)} .
$$

To estimate the second term $\theta_{h}(t)$, we set $\Phi_{h}=\left(v_{h}, w_{h}\right)$ and note that

$$
\begin{aligned}
c_{h}\left(\partial_{t} \theta_{h}(t) ; \Phi_{h}\right)+a_{h}\left(\theta_{h}(t) ; \Phi_{h}\right) & \\
& =c_{h}\left(\partial_{t} U_{h}(t) ; \Phi_{h}\right)-c_{h}\left(R_{h} \partial_{t} U(t) ; \Phi_{h}\right)+a_{h}\left(U_{h}(t) ; \Phi_{h}\right)-a_{h}\left(R_{h} U(t) ; \Phi_{h}\right) \\
& =\widetilde{c}\left(\partial_{t} \widetilde{U}(t) ; \Phi_{h}\right)-c_{h}\left(R_{h} \partial_{t} U(t) ; \Phi_{h}\right)+\widetilde{a}\left(\widetilde{U}(t) ; \Phi_{h}\right)-a_{h}\left(R_{h} U(t) ; \Phi_{h}\right) .
\end{aligned}
$$


Here we used the variational characterizations (23) and (24) of the discrete and the continuous solution. By definition (34) of the elliptic projection, we further get

$$
\begin{aligned}
& c_{h}\left(\partial_{t} \theta_{h}(t) ; \Phi_{h}\right)+a_{h}\left(\theta_{h}(t) ; \Phi_{h}\right) \\
& =\widetilde{c}\left(\partial_{t} \widetilde{U}(t) ; \Phi_{h}\right)-c_{h}\left(R_{h} \partial_{t} U(t) ; \Phi_{h}\right)-\eta c_{h}\left(\widetilde{U}(t) ; \Phi_{h}\right)+\eta c_{h}\left(R_{h} U(t) ; \Phi_{h}\right) \\
& =\left[\widetilde{c}\left(\partial_{t} \widetilde{U}(t) ; \Phi_{h}\right)-c_{h}\left(\partial_{t} \widetilde{U}(t) ; \Phi_{h}\right)\right]+c_{h}\left(\partial_{t} \widetilde{U}(t)-R_{h} \partial_{t} U(t) ; \Phi_{h}\right)+\eta c_{h}\left(R_{h} U(t)-\widetilde{U}(t) ; \Phi_{h}\right) .
\end{aligned}
$$

Using Lemma 19 to bound the first term, the estimates for the elliptic projection given in Lemma 28, and the Cauchy-Schwarz inequality, we obtain

$$
\begin{aligned}
c_{h}\left(\partial_{t} \theta_{h}(t) ;\right. & \left.\Phi_{h}\right)+a_{h}\left(\theta_{h}(t) ; \Phi_{h}\right) \\
& \leq C h^{2}\left(\|U(t)\|_{H^{2}(\Omega) \times H^{2}(\Gamma)}+\left\|\partial_{t} U(t)\right\|_{H^{2}(\Omega) \times H^{2}(\Gamma)}\right)\left\|\Phi_{h}\right\|_{H^{1}\left(\Omega_{h}\right) \times H^{1}\left(\Gamma_{h}\right)} .
\end{aligned}
$$

We now choose $\Phi_{h}=\left(v_{h}, w_{h}\right)$ with $v_{h}=\lambda\left(L_{h}(t)-\widetilde{L}_{R}(t)\right)$ and $w_{h}=\gamma\left(\ell_{h}(t)-\widetilde{\ell}_{R}(t)\right)$ as the test function and define $E_{h}(\theta(t))=\frac{1}{2}\|\theta(t)\|_{L^{2}\left(\Omega_{h}\right) \times L^{2}\left(\Gamma_{h}\right)}^{2}$ and $D_{h}\left(\theta_{h}(t)\right)=a_{h}\left(\theta_{h}(t) ; \Phi_{h}\right)$. With the previous estimates and the same reasoning as in Lemma 25 and 26, we get

$$
\begin{aligned}
& \frac{d}{d t} E_{h}\left(\theta_{h}(t)\right)+D_{h}\left(\theta_{h}(t)\right)=c_{h}\left(\partial_{t} \theta_{h}(t), \Phi_{h}\right)+a_{h}\left(\theta_{h}(t) ; \Phi_{h}\right) \\
& \quad \leq C^{\prime} h^{2}\left(\left\|\partial_{t} U(t)\right\|_{H^{2}(\Omega) \times H^{2}(\Gamma)}+\|U(t)\|_{H^{2}(\Omega) \times H^{2}(\Gamma)}\right)\left\|\theta_{h}(t)\right\|_{H^{1}\left(\Omega_{h}\right) \times H^{1}\left(\Gamma_{h}\right)} .
\end{aligned}
$$

We also used here that the norms of $\Phi_{h}$ and $\theta_{h}(t)$ are comparable by construction. By Lemma 28 the total mass of $\theta_{h}(t)=U_{h}(t)-R_{h} U(t)$ is zero, and we conclude from Lemma 22 that

$$
D_{h}\left(\theta_{h}\right) \geq c\left\|\theta_{h}\right\|_{H^{1}\left(\Omega_{h}\right) \times H^{1}\left(\Gamma_{h}\right)}^{2} \geq c^{\prime} E_{h}(\theta) .
$$

Thus we can absorb the term $\left\|\theta_{h}(t)\right\|_{H^{1}\left(\Omega^{h}\right) \times H^{1}\left(\Gamma_{h}\right)}$ in the above estimate via Young's inequality by the entropy dissipation $D_{h}\left(\theta_{h}(t)\right)$ on the left hand side which finally leads to

$$
\frac{d}{d t} E_{h}(\theta(t))+c_{1} E_{h}(\theta(t)) \leq C^{\prime \prime} h^{4}\left(\left\|\partial_{t} U(t)\right\|_{H^{2}(\Omega) \times H^{2}(\Gamma)}^{2}+\|U(t)\|_{H^{2}(\Omega) \times H^{2}(\Gamma)}^{2}\right)
$$

The claim now follows by application of the Gronwall inequality and noting that the initial conditions are approximated with optimal order.

\section{Time Discretization}

As a final step towards the numerical solution, let us now turn to the time discretization. For ease of notation, we only consider the backward Euler scheme in detail here. After introducing the scheme and the relevant notation, we again establish the convergence to equilibrium and prove convergence estimates that are uniform in time.

5.1. Implicit backward Euler scheme. Let $\tau>0$ be fixed and define $t^{n}=n \tau$ for all $n=0,1,2, \ldots$. Given a sequence $\left\{u^{n}\right\}_{n \geq 0}$, we denote by

$$
\bar{\partial}_{\tau} u^{n}:=\frac{u^{n}-u^{n-1}}{\tau}
$$

the backward difference quotient which serves as the approximation for the time derivative. Our fully discrete approximation for the system (3a)-(3d) then reads

Problem 30 (Time discretization). Define $\left(L_{h}^{0}, \ell_{h}^{0}\right) \in V_{h} \times W_{h}$ by

$$
\left(L_{h}^{0}, v_{h}\right)_{\Omega_{h}}=\left(\widetilde{L}^{0}, v_{h}\right)_{\Omega_{h}} \quad \text { and } \quad\left(\ell_{h}^{0}, w_{h}\right)_{\Gamma_{h}}=\left(\widetilde{\ell}^{0}, w_{h}\right)_{\Gamma_{h}}
$$

for all $\left(v_{h}, w_{h}\right) \in V_{h} \times W_{h}$. Then for $n=1,2, \ldots$, find $\left(L_{h}^{n}, \ell_{h}^{n}\right) \in V_{h} \times W_{h}$ such that

$$
c_{h}\left(\bar{\partial}_{\tau} L_{h}^{n}, \bar{\partial}_{\tau} \ell_{h}^{n} ; v_{h}, w_{h}\right)+a_{h}\left(L_{h}^{n}, \ell_{h}^{n} ; v_{h}, w_{h}\right)=0
$$

for all test functions $\left(v_{h}, w_{h}\right) \in V_{h} \times W_{h}$. 
The problems (37) for the individual time steps can be written equivalently as

$$
\frac{1}{\tau} c_{h}\left(L_{h}^{n}, \ell_{h}^{n} ; v_{h}, w_{h}\right)+a_{h}\left(L_{h}^{n}, \ell_{h}^{n} ; v_{h}, w_{h}\right)=\frac{1}{\tau} c_{h}\left(L_{h}^{n-1}, \ell_{h}^{n-1} ; v_{h}, w_{h}\right) .
$$

From this representation and the discrete inf-sup stability condition (27), one can directly deduce that the problems (37) are uniquely solvable.

Lemma 31 (Well-posedness). Let $\left(L_{h}^{n-1}, \ell_{h}^{n-1}\right) \in L^{2}\left(\Omega_{h}\right) \times L^{2}\left(\Gamma_{h}\right)$ be given. Then for any $\tau>0$, Problem 30 admits a unique solution $\left(L_{h}^{n}, \ell_{h}^{n}\right) \in V_{h} \times W_{h}$.

Problem 30 therefore defines a sequence $\left\{L_{h}^{n}, \ell_{h}^{n}\right\}_{n \geq 0} \subset V_{h} \times W_{h}$ which we call the fully discrete solution. By testing with $v_{h} \equiv 1$ and $w_{h} \equiv 1$, we further obtain

Lemma 32 (Mass conservation). Let $M_{h}^{0}:=\left(\widetilde{L}^{0}, 1\right)_{\Omega}+\left(\widetilde{\ell}^{0}, 1\right)_{\Gamma_{h}}$. Then,

$$
\left(L_{h}^{n}, 1\right)_{\Omega_{h}}+\left(\ell_{h}^{n}, 1\right)_{\Gamma_{h}}=M_{h}^{0} \quad \text { for all } n \geq 1,
$$

i.e., the total mass is conserved for all time steps.

Remark 33. By the usual mass lumping and under additional assumptions on the mesh, also non-negativity of solutions could be guaranteed for all time steps provided that the initial conditions were non-negative; let us again refer to [5, 25] for details.

5.2. Convergence to the discrete equilibrium. To study the large time behavior of the discrete evolution, we utilize the discrete entropy functional defined in Section 4 given by

$$
E_{h}(L, \ell)=\frac{1}{2}\left(\lambda\left\|L-L_{h}^{\infty}\right\|_{\Omega_{h}}^{2}+\gamma\left\|\ell-\ell_{h}^{\infty}\right\|_{\Gamma_{h}}^{2}\right) .
$$

As a replacement for the entropy dissipation derived in Lemma 25, we now have

Lemma 34 (Fully discrete entropy dissipation). For all $n \geq 1$, there holds

$$
\begin{aligned}
\bar{\partial}_{\tau} E_{h}\left(L_{h}^{n}, \ell_{h}^{n}\right) \leq-\lambda d_{L} \| \nabla & \left(L_{h}^{n}-L_{h}^{\infty}\right)\left\|_{\Omega_{h}}^{2}-\gamma d_{\ell}\right\| \nabla_{\Gamma}\left(\ell_{h}^{n}-\ell_{h}^{\infty}\right) \|_{\Gamma_{h}}^{2} \\
& -\left\|\lambda\left(L_{h}^{n}-L_{h}^{\infty}\right)-\gamma\left(\ell_{h}^{n}-\ell_{h}^{\infty}\right)\right\|_{\Gamma}^{2}=-D_{h}\left(L_{h}^{n}, \ell_{h}^{n}\right) .
\end{aligned}
$$

Proof. One can verify by direct computation that

$$
\bar{\partial}_{\tau} E_{h}\left(L_{h}^{n}, \ell_{h}^{n}\right) \leq\left(\bar{\partial}_{\tau} L_{h}^{n}, \lambda\left(L_{h}^{n}-L_{h}^{\infty}\right)\right)_{\Omega_{h}}+\left(\bar{\partial}_{\tau} \ell_{h}^{n}, \gamma\left(\ell_{h}^{n}-\ell_{h}^{\infty}\right)\right)_{\Gamma_{h}} .
$$

The assertion then follows along the lines of the proof of Lemma 25.

The exponential convergence to equilibrium can now be established in a similar manner as on the semi-discrete level by using the previous lemma, the entropy-entropy dissipation inequality of Lemma 26, and a discrete Gronwall inequality. Summarizing, we obtain

Theorem 35 (Convergence to the discrete equilibrium). For any $\tau>0$ and $n \geq 0$, there holds

$$
\left\|L_{h}^{n}-L_{h}^{\infty}\right\|_{\Omega_{h}}^{2}+\left\|\ell_{h}^{n}-\ell_{h}^{\infty}\right\|_{\Gamma_{h}}^{2} \leq C e^{-c_{0} \tau n}\left(\left\|L_{h}^{0}-L_{h}^{\infty}\right\|_{\Omega_{h}}^{2}+\left\|\ell_{h}^{0}-\ell_{h}^{\infty}\right\|_{\Gamma_{h}}^{2}\right) .
$$

The constants $C$ and $c_{0}$ can be chosen the same as in Theorem $2 \%$.

Let us emphasize that the constants are independent of the discretization parameters and the time horizon. This will allow us to establish uniform bounds for the discretization error. 
5.3. Error estimates for the full discretization. For the error analysis on the fully discrete level, we again slightly extend standard arguments $[25,26]$ by appropriately taking into account the geometric errors. Our main error estimate for the full discretization reads

Theorem 36 (Error estimates for the full discretization). Let (A1)-(A3) hold and assume that the solution $(L, \ell)$ of $(3 \mathrm{a})-(3 \mathrm{~d})$ is sufficiently smooth. Then for all $n \geq 0$ we have

$$
\left\|L_{h}^{n}-\widetilde{L}\left(t^{n}\right)\right\|_{\Omega_{h}}+\left\|\ell_{h}^{n}-\widetilde{\ell}\left(t^{n}\right)\right\|_{\Gamma_{h}} \leq C\left(h^{2}+\tau\right)
$$

with a constant $C$ that is independent of the discretization parameters $\tau$ and $h$ and of $n$.

Proof. For ease of notation, we again write $U_{h}^{n}=\left(L_{h}^{n}, \ell_{h}^{n}\right), \widetilde{U}=(\widetilde{L}, \widetilde{\ell})$, and $R_{h} U=\left(\widetilde{L}_{R}, \widetilde{\ell}_{R}\right)$. Using the elliptic projection, the error can be decomposed into

$$
U_{h}^{n}-\widetilde{U}\left(t^{n}\right)=\left[U_{h}^{n}-R_{h} U\left(t^{n}\right)\right]+\left[R_{h} U\left(t^{n}\right)-\widetilde{U}\left(t^{n}\right)\right]=: \theta_{h}^{n}+\rho^{n} .
$$

By the properties of the operator $R_{h}$, the first error component $\rho^{n}$ can be estimated by

$$
\left\|\rho^{n}\right\|_{L^{2}\left(\Omega_{h}\right) \times L^{2}\left(\Gamma_{h}\right)} \leq C h^{2}\left\|U\left(t^{n}\right)\right\|_{H^{2}(\Omega) \times H^{2}(\Gamma)} .
$$

Similar as in the proof of Theorem 29, we can characterize the second error component by

$$
\begin{aligned}
& c_{h}\left(\bar{\partial}_{\tau} \theta_{h}^{n}, \Phi_{h}\right)+a_{h}\left(\theta_{h}^{n} ; \Phi_{h}\right) \\
& \quad=c_{h}\left(\bar{\partial}_{\tau} U_{h}^{n}, \Phi_{h}\right)-c_{h}\left(\bar{\partial}_{\tau} R_{h} U\left(t^{n}\right), \Phi_{h}\right)+a_{h}\left(U_{h}^{n} ; \Phi_{h}\right)-a_{h}\left(R_{h} U\left(t^{n}\right) ; \Phi_{h}\right) \\
& =\left[\widetilde{c}\left(\bar{\partial}_{\tau} \widetilde{U}\left(t^{n}\right), \Phi_{h}\right)-c_{h}\left(\bar{\partial}_{\tau} \widetilde{U}\left(t^{n}\right) ; \Phi_{h}\right)\right]+c_{h}\left(\bar{\partial}_{\tau} \widetilde{U}\left(t^{n}\right)-R_{h} \bar{\partial}_{\tau} U\left(t^{n}\right) ; \Phi_{h}\right) \\
& \quad \quad+\eta c_{h}\left(R_{h} U\left(t^{n}\right)-\widetilde{U}\left(t^{n}\right) ; \Phi_{h}\right)+\widetilde{c}\left(\partial_{t} \widetilde{U}\left(t^{n}\right)-\bar{\partial}_{\tau} \widetilde{U}\left(t^{n}\right) ; \Phi_{h}\right) \\
& \quad \\
& =(i)+(i i)+(i i i)+(i v) .
\end{aligned}
$$

The first three terms can be estimated as in Theorem 29, and for the fourth term, we use

$$
\bar{\partial}_{\tau} \widetilde{U}\left(t^{n}\right)-\partial_{t} \widetilde{U}\left(t^{n}\right)=-\int_{t^{n-1}}^{t^{n}} \int_{t}^{t^{n}} U_{t t}(s) d s d t
$$

Proceeding similarly as in the proof of Theorem 29, we then arrive at

$$
\begin{aligned}
& \bar{\partial}_{\tau} E_{h}\left(\theta_{h}^{n}\right)+c_{1} E_{h}\left(\theta_{h}^{n}\right) \\
& \quad \leq C^{\prime}\left(h^{4}\left\|\partial_{t} U\left(t^{n}\right)\right\|_{H^{2}\left(\Omega \times H^{2}(\Omega)\right.}^{2}+h^{4}\left\|U\left(t^{n}\right)\right\|_{H^{2}(\Omega) \times H^{2}(\Omega)}^{2}+\tau^{2}\left\|U_{t t}\left(\xi^{n}\right)\right\|_{L^{2}(\Omega) \times L^{2}(\Gamma)}^{2}\right)
\end{aligned}
$$

for some positive constants $c_{1}, C^{\prime}>0$ and appropriate $\xi^{n} \in\left(t^{n-1}, t^{n}\right)$. The result now follows by a discrete Gronwall lemma.

Let us emphasize once more that all error estimates hold uniformly in time. By simple inspection of the proof, the precise regularity requirements can be made explicit.

\section{NuMERICS}

For illustration of our theoretical results, we now report about some numerical results obtained for the model problem (1a)-(1c). For ease of presentation, we restrict ourselves to two space dimensions and consider $\Omega$ to be the unit circle. In the simulations, we chose the model parameters as $d_{L}=0.01, d_{\ell}=0.02, \gamma=2$, and $\lambda=4$, and we set the initial conditions to

$$
L^{0}(x, y)=\frac{1}{2}\left(x^{2}+y^{2}\right) \quad \text { and } \quad \ell^{0}(x, y)=\frac{1}{2}(1+x) .
$$

We start by investigating the convergence of solutions with respect to the mesh size. To this end, we generate a sequence of meshes $\left\{T_{h}\right\}_{h>0}$ by uniform refinement of an initial triangulation consisting of 258 elements. We further choose $T=2$ as the final time and $\tau=0.01$ as the time 
step size. As a computable approximation for the error at time $t=T$, we use the difference of solutions obtained on two consecutive refinements. To be precise, we use

$$
L(T)-L_{h}^{N}(T) \approx L_{h / 2}^{N}-L_{h}^{N}:=\triangle L_{h}
$$

and $\triangle \ell_{h}=\ell_{h / 2}^{N}-\ell_{h}^{N}$ to measure the error for the error in the volume and surface concentrations, respectively. The results obtained in our numerical tests are displayed in Table 6 . They confirm

\begin{tabular}{c||c|c||c|c||c||c||c|c}
$h$ & $\left\|\triangle L_{h}\right\|_{L^{2}}$ & rate & $\left\|\triangle \ell_{h}\right\|_{L^{2}}$ & rate & $\left\|\triangle L_{h}\right\|_{H^{1}}$ & rate & $\left\|\triangle \ell_{h}\right\|_{H^{1}}$ & rate \\
\hline 0.2500 & 0.0066 & - & 0.0029 & - & 0.1193 & - & 0.0390 & - \\
0.1250 & 0.0018 & 1.88 & 0.0007 & 1.98 & 0.0608 & 0.97 & 0.0195 & 1.00 \\
0.0625 & 0.0005 & 1.96 & 0.0002 & 1.99 & 0.0306 & 0.99 & 0.0097 & 1.00 \\
0.0313 & 0.0001 & 1.99 & 0.0000 & 2.01 & 0.0154 & 1.00 & 0.0049 & 1.00 \\
0.0016 & 0.0000 & 2.00 & 0.0000 & 1.94 & 0.0077 & 1.00 & 0.0024 & 1.00
\end{tabular}

TABLE 1. Errors obtained on a sequence of uniformly refined meshes for the model problem (1a)-(1c) with convergence rate estimated from two consecutive levels.

the convergence rates that were predicted by Theorem 29. Let us mention that the $O(h)$ convergence of the error in the $H^{1}$-norm can also be explained theoretically $[25,26]$.

In a second test, we verify the convergence with respect to the time step size $\tau$. For that we use a spatially refined mesh $T_{h}$ with mesh size $h=0.03125$. As before, we set the final time to $T=2$ and we run the simulations for different time steps sizes $\tau=2^{-k}, k=1,2, \ldots$ The results obtained in our simulations are depicted in Table 6 .

\begin{tabular}{c||c|c||c|c||c||c||c|c}
$\tau$ & $\left\|\triangle L_{h}\right\|_{L^{2}}$ & rate & $\left\|\triangle \ell_{h}\right\|_{L^{2}}$ & rate & $\left\|\triangle L_{h}\right\|_{H^{1}}$ & rate & $\left\|\triangle \ell_{h}\right\|_{H^{1}}$ & rate \\
\hline 0.5000 & 0.0082 & - & 0.0109 & - & 0.0464 & - & 0.0154 & - \\
0.2500 & 0.0046 & 0.82 & 0.0057 & 0.94 & 0.0269 & 0.79 & 0.0080 & 0.94 \\
0.1250 & 0.0025 & 0.90 & 0.0029 & 0.97 & 0.0147 & 0.88 & 0.0041 & 0.97 \\
0.0625 & 0.0013 & 0.95 & 0.0015 & 0.98 & 0.0077 & 0.93 & 0.0021 & 0.98 \\
0.0313 & 0.0007 & 0.97 & 0.0007 & 0.99 & 0.0039 & 0.96 & 0.0010 & 0.99
\end{tabular}

TABLE 2. Errors and convergence rates obtained with decreasing time step size $\tau$ for the model problem (1a)-(1c) with rate computed from two consecutive levels.

The error history clearly indicates convergence of first order with respect to $\tau$ which agrees with the rate predicted by Theorem 36 . Note that the same convergence rate is observed for the error in the $L^{2}$ - and the $H^{1}$-norm. Also this observation could be proven theoretically.

With the third test, we would like to illustrate the large time behavior of the system. To this end, we choose $T=500$ and $\tau=0.5$. We then compute the discrete solutions for a sequence of uniformly refined meshes and evaluate the entropy, to be more precise, we compute

$$
\widetilde{E}\left(L_{h}^{N}, \ell_{h}^{N}\right)=\frac{1}{2}\left(\lambda\left\|L_{h}^{N}-L^{\infty}\right\|_{\Omega_{h}}^{2}+\gamma\left\|\ell_{h}^{N}-\ell^{\infty}\right\|_{\Gamma_{h}}^{2}\right),
$$

which measures the distance of the discrete solution to the exact equilibrium state. This allows us to evaluate at the same time the convergence to equilibrium and the inexact approximation of the equilibrium state which is due to the geometric errors. The corresponding results are displayed in Figure 2. As predicted by our theoretical results, we observe uniform exponential convergence to equilibrium. The numerical results also allow us to estimate the exponential 


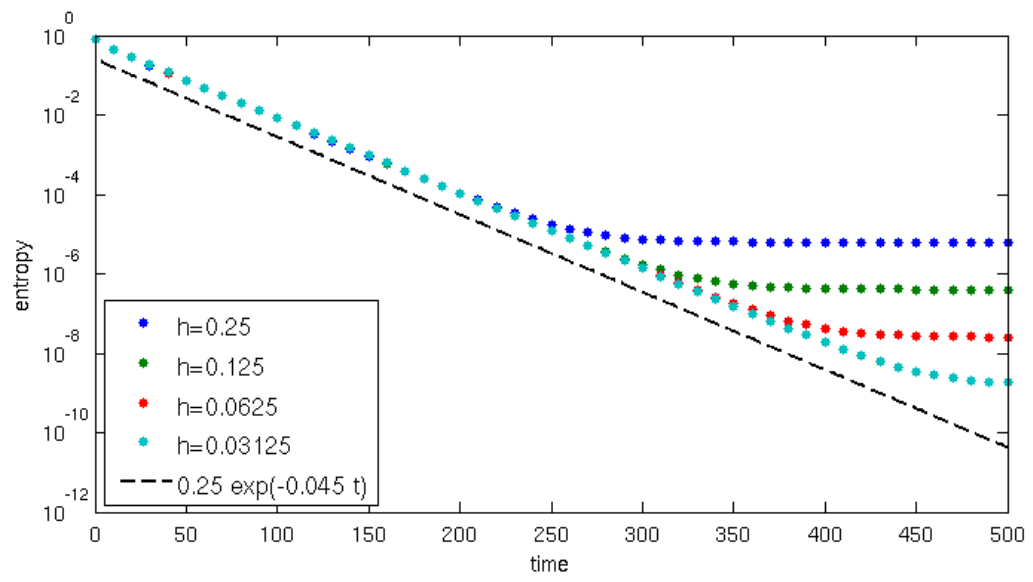

FiguRE 2. Decay of the entropy $\widetilde{E}\left(L_{h}\left(t^{n}\right), \ell_{h}\left(t^{n}\right)\right)$ over time. One can clearly see the exponential convergence with a mesh independent rate. Note that for large time, the discretization error for the equilibrium state becomes dominant and leads to saturation.

decay rate which is approximately $c_{0}=0.045$ here. For large time, the discretization errors due to approximation of the equilibrium becomes dominant; compare with Lemma 24.

\section{Extension to A SYSTem ARISING IN ASYMmetriC STEM CELL DIVISION}

As a final step of our considerations, we would like to illustrate now that the analysis and the discretization strategy presented for the model problem (3a)-(3c) can be extended with minor modifications to more general volume-surface reaction-diffusion systems which share the same key properties: (i) non-negativity of solutions, (ii) conservation of mass, (iii) a constant positive detailed balance equilibrium, and (iv) a quadratic entropy functional and an appropriate entropy-entropy dissipation estimate.

As an example, we consider the following system describing the evolution of two volume and two surface concentrations, which was already mentioned briefly in the introduction.

$$
\begin{aligned}
\partial_{t} L-d_{L} \Delta L=-\beta L+\alpha P, & x \in \Omega, t>0, \\
\partial_{t} P-d_{P} \Delta P=\beta L-\alpha P, & x \in \Omega, t>0, \\
\partial_{t} \ell-d_{\ell} \Delta_{\Gamma} \ell=-d_{L} \partial_{n} L+\chi_{\Gamma_{2}}(-\sigma \ell+\kappa p), & x \in \Gamma, t>0, \\
\partial_{t} p-d_{p} \Delta_{\Gamma_{2}}=\sigma \ell-\kappa p-d_{P} \partial_{n} P, & x \in \Gamma_{2}, t>0 .
\end{aligned}
$$

As before, $\Omega$ is a bounded domain in two or three dimensions with a smooth boundary $\Gamma=\partial \Omega$. Furthermore, $\Gamma_{2} \subset \Gamma$ is a proper subpart with smooth boundary $\partial \Gamma_{2}$. We denote by $\chi_{\Gamma_{2}}$ the characteristic function on $\Gamma_{2}$. The reactions and the mass transfer between the volume and the surface are governed by

$$
\begin{aligned}
d_{L} \partial_{n} L=-\lambda L+\gamma \ell, & x \in \Gamma, t>0, \\
d_{P} \partial_{n} P=\chi_{\Gamma_{2}}(-\eta P+\xi p), & x \in \Gamma, t>0, \\
d_{p} \partial_{n_{\Gamma}} p=0, & x \in \partial \Gamma_{2}, t>0,
\end{aligned}
$$

and the system is complemented by appropriate initial conditions. A schematic sketch of the reaction dynamics is given in Figure 1.

A variant of the system (45a)-(45g) was studied recently in [15] as a model for the asymmetric localization of Lgl during the mitosis of SOP stem cells of Drosophila [2, 20, 27]. The volume 
concentrations $L, P$ then represent the cytoplasmic pure and phosphorylated form of the protein Lgl (Lethal giant larvae) which is essential in triggering the asymmetric localization of socalled cell-fate determinant proteins during asymmetric division. Moreover, $\ell, p$ represent the corresponding cortical Lgl concentration. A detailed discussion can be found in [15].

We assume that all model parameters are positive constants and that the system has a detailed balance equilibrium, i.e. we require that

$$
\frac{\alpha \lambda \sigma \xi}{\beta \gamma \kappa \eta}=1 .
$$

Using this condition, one can show that the system (45a)-(45g) has very similar properties as the model problem (3a)-(3c) and, therefore, the analysis of the previous sections can be carried over to the system (45) almost verbatim. Let us sketch the necessary key steps in more detail:

Step 1. The system (45a)-(45g) has an inherent mass conservation law, i.e.,

$$
M(t):=\int_{\Omega} L(t)+P(t) d x+\int_{\Gamma} \ell(t) d s+\int_{\Gamma_{2}} p(t) d s=M(0) \quad \text { for all } t>0 .
$$

Step 2. Together with the detailed balance condition (46), one can show that for any initial mass $M^{0}>0$ there exists a unique positive constant equilibrium $\left(L^{\infty}, P^{\infty}, \ell^{\infty}, p^{\infty}\right)$. Again, analytic formulas depending only on the initial mass $M^{0}$, on the model parameters, and on the geometry can be derived.

Step 3. We can define a quadratic relative entropy functional, which here has the form

$$
\begin{aligned}
E(L, P, \ell, p)=\frac{1}{2}\left(\int_{\Omega} \frac{1}{L^{\infty}}\left|L-L^{\infty}\right|^{2} d x+\int_{\Omega} \frac{1}{P^{\infty}}\left|P-P^{\infty}\right|^{2} d x\right. \\
\left.+\int_{\Gamma} \frac{1}{\ell^{\infty}}\left|\ell-\ell^{\infty}\right|^{2} d s+\int_{\Gamma_{2}} \frac{1}{p^{\infty}}\left|p-p^{\infty}\right|^{2} d s\right) .
\end{aligned}
$$

Let us mention that, up to scaling with a constant, also the entropy functional for the model problem (3a)-(3c) defined in (13) could be written in this way.

Step 4. The corresponding entropy dissipation relation now reads

$$
\begin{aligned}
\frac{d}{d t} & E(L(t), P(t), \ell(t), p(t)) \\
= & -\frac{d_{L}}{L^{\infty}}\|\nabla L(t)\|_{\Omega}^{2}-\frac{d_{P}}{P^{\infty}}\|\nabla P(t)\|_{\Omega}-\frac{d_{\ell}}{\ell^{\infty}}\left\|\left.\nabla_{\Gamma} \ell(t)\right|_{\Gamma} ^{2}-\frac{d_{p}}{p^{\infty}}\right\| \nabla_{\Gamma_{2}} p(t) \|_{\Gamma_{2}}^{2} \\
& \left.-\frac{1}{\beta L^{\infty}}\|\beta L(t)-\alpha P(t)\|_{\Omega}^{2}-\frac{1}{\gamma \ell^{\infty}} \| \gamma \ell(t)-\lambda L(t)\right)^{2} \|_{\Gamma}^{2} \\
& -\frac{1}{\kappa p^{\infty}}\|\kappa p(t)-\sigma \ell(t)\|_{\Gamma_{2}}^{2}-\frac{1}{\eta P^{\infty}}\|\eta P(t)-\xi p(t)\|_{\Gamma_{2}}^{2}=:-D(L(t), P(t), \ell(t), p(t)) .
\end{aligned}
$$

Step 5. Similar as in Lemma 7, an entropy-entropy dissipation estimate of the form

$$
D(L, P, \ell, p) \geq c_{0} E(L, P, \ell, p)
$$

holds with a constant $c_{0}$ only depending on the model parameters and on the domain. Similar as above, the proof of this estimate is again based on a Poincaré-type inequality.

Following the arguments of Sections $2-5$ one can then establish the following results:

Result 1. Convergence to equilibrium for the continuous problem, i.e.,

$$
\left\|L(t)-L^{\infty}\right\|_{\Omega}+\left\|P(t)-P^{\infty}\right\|_{\Omega}+\left\|\ell(t)-\ell^{\infty}\right\|_{\Gamma}+\left\|p(t)-p^{\infty}\right\|_{\Gamma_{2}} \leq C e^{-c_{0} t} \text { for all } t>0 .
$$

Result 2. Convergence to the discrete equilibrium for semi-discrete solutions

$$
\left\|L_{h}(t)-L_{h}^{\infty}\right\|_{\Omega_{h}}+\left\|P_{h}(t)-P_{h}^{\infty}\right\|_{\Omega_{h}}+\left\|\ell_{h}(t)-\ell_{h}^{\infty}\right\|_{\Gamma_{h}}+\left\|p_{h}(t)-p_{h}^{\infty}\right\|_{\Gamma_{2, h}} \leq C e^{-c_{0} t},
$$

with constants $c_{0}, C$ independent of the mesh size $h>0$ 
Result 3. Convergence for the fully discrete solution, i.e.,

$$
\left\|L_{h}^{n}-L_{h}^{\infty}\right\|_{\Omega_{h}}+\left\|P_{h}^{n}-P_{h}^{\infty}\right\|_{\Omega_{h}}+\left\|\ell_{h}^{n}-\ell_{h}^{\infty}\right\|_{\Gamma_{h}}+\left\|p_{h}^{n}-p_{h}^{\infty}\right\|_{\Gamma_{2, h}} \leq C e^{-c_{0} \tau n},
$$

with constants $c_{0}, C$ independent of the mesh size $h>0$ and the time step $\tau>0$.

Result 4. Error estimates and order optimal convergence for the semi-discretization independent of time horizon

$$
\left\|L_{h}(t)-\widetilde{L}(t)\right\|_{\Omega_{h}}+\left\|P_{h}(t)-\widetilde{P}(t)\right\|_{\Omega_{h}}+\left\|\ell_{h}(t)-\widetilde{\ell}(t)\right\|_{\Gamma_{h}}+\left\|p_{h}(t)-\widetilde{p}(t)\right\|_{\Gamma_{2, h}} \leq C h^{2} .
$$

Result 5. Order optimal error estimates for the full discretization

$$
\left\|L_{h}^{n}-\widetilde{L}\left(t^{n}\right)\right\|_{\Omega_{h}}+\left\|P_{h}^{n}-\widetilde{P}\left(t^{n}\right)\right\|_{\Omega_{h}}+\left\|\ell_{h}^{n}-\widetilde{\ell}\left(t^{n}\right)\right\|_{\Gamma_{h}}+\left\|p_{h}^{n}-\widetilde{p}\left(t^{n}\right)\right\|_{\Gamma_{2, h}} \leq C\left(h^{2}+\tau\right)
$$

with constant $C$ that is independent of the time horizon, of the mesh size, and of the time step.

Conclusion: All results obtained in the previous sections for the model problem (3a)-(3d) carry over almost verbatim to more complex problems sharing the same key properties.

For illustration of the solution behavior, some snapshots of the concentrations $P(t)$ and $L(t)$ for a typical scenario are depicted in Figure 7.
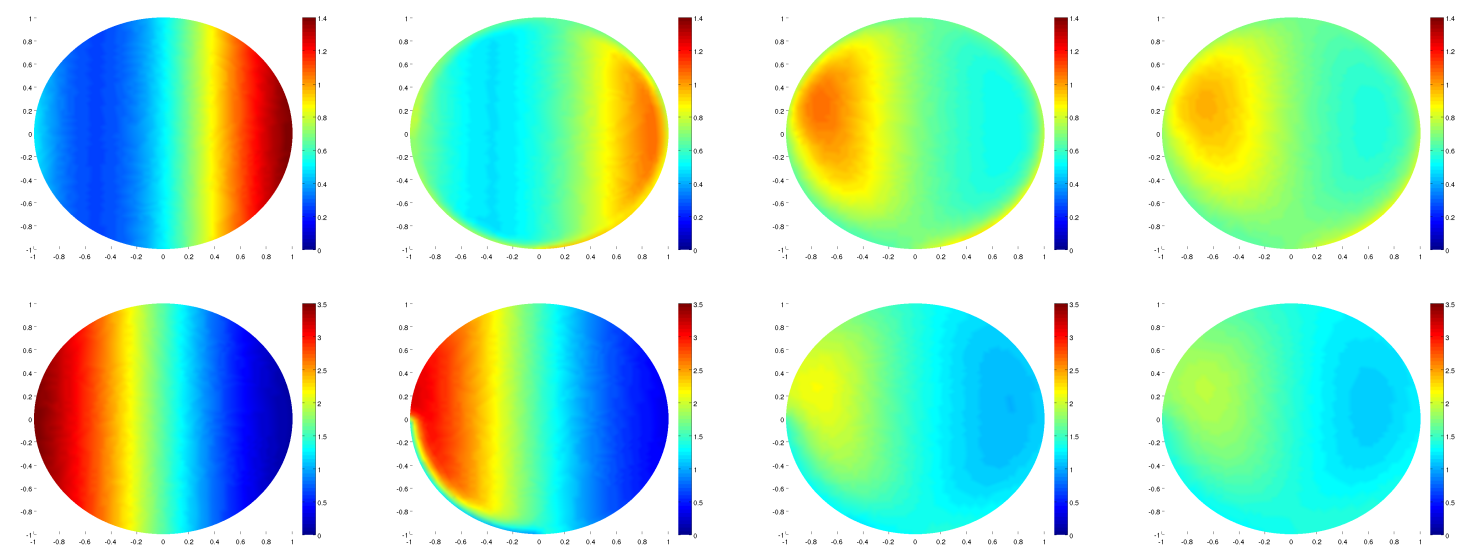

Figure 3. Snapshots for $L(t)$ and $P(t)$ at $t=0,0.13,1.56,3.0$. A mesh with 4064 triangles and $\tau=0.01$ was used. The initial data were $L^{0}(x, y)=x \sin (x+1)+$ $0.5, P^{0}(x, y)=(2-x) \cos (x+1)+0.5, \ell^{0}(x, y)=0.3(2-y)+1$, and $p^{0}(x, y)=0.4 y+1$.

As can be seen from the images, the evolution is driven by convergence to the constant equilibrium. Also some local effects due to the mass transfer with the boundary can be observed.

\section{SUMMARY}

Volume-surface reaction-diffusion systems arise in many applications in chemistry, in fluid dynamics, in crystal growth [17, 22], and also in molecular-biology, where many current models aim to describe signaling pathways [16] or the evolution of proteins [15]. Realistic models often involve several species and the mathematical analysis becomes increasingly cumbersome for larger systems. The guideline of this paper therefore was to develop analytical and numerical methods which are general in the sense that they are based only on a few fundamental properties which are shared by many problems, e.g., the non-negativity of solutions, constant equilibria, exponential stability and convergence to equilibrium. 
Although we confined ourselves here to a simple model problem, our arguments, in particular the use of entropy estimates for the analysis on the continuous and on the discrete level, can be used to obtain similar results for a large class of surface-volume reaction-diffusion problems having constant equilibrium states. Big parts of our analysis could even be extended to problems with non-constant equilibria. In particular, quadratic entropy functionals of the form (48) can be constructed for a wide class of problems with linear reaction dynamics. In the general situation, the entropies can however not be used so easily on the discrete level. Another direction of further research are complex balanced systems, e.g., weakly reversible reaction networks. Such systems still feature positive equilibria and exponential convergence to equilibrium, as recently established for general first order reaction-diffusion networks in [14]. The formulation of an appropriate discrete entropy functional and the corresponding entropy dissipation estimate however require some non-trivial modifications of the arguments and are topics of current research.

Acknowledgments. This work was partly carried out during the visits of the second and the last author to TU Darmstadt. KF and BT gratefully acknowledge the hospitality of TU Darmstadt and financial support by IRTG 1529, IRTG 1754, and NAWI Graz. HE was supported by DFG via grants IRTG 1529, GSC 233, and TRR 154. The work of JFP was supported by DFG via Grant 1073/1-1, by the Daimler and Benz Stiftung via Post-Doc Stipend 32-09/12 and by the German Academic Exchange Service via PPP grant No. 56052884.

\section{REFERENCES}

[1] E. Bänsch and K. Deckelnick. Optimal error estimates for the Stokes and Navier-Stokes equations with slip-boundary condition. Math. Model. Numer. Anal. 33 (1999) 923-938.

[2] J. Betschinger, K. Mechtler, and J. A. Knoblich. The Par complex directs asymmetric cell division by phosphorylating the cytoskeletal protein Lgl. Nature 422 (2003) 326-329.

[3] M. Bukal, E. Emmrich, and A. Jüngel. Entropy-stable and entropy-dissipative approximations of a fourthorder quantum diffusion equation. Numer. Math. 127 (2014), 365-396

[4] C. Cancès and C. Guichard. Numerical analysis of a robust entropy-diminishing finite-volume scheme for parabolic equations with gradient structure. Preprint, 2015. arXiv:1503.05649.

[5] C. M. Chen and V. Thomee. The lumped mass finite element method for a parabolic problem. J. Austral. Math. Soc. Ser. B 26 (1985) 329-354.

[6] C. Chainais-Hillairet, A. Jüngel, and S. Schuchnigg. Entropy-dissipative discretization of nonlinear diffusion equations and discrete Beckner inequalities. To appear in ESAIM Math. Model. Numer. Anal. 2015, arXiv:1303.3791.

[7] S. Christiansen, H. Munthe-Kaas, and B. Owren. Topics in structure-preserving discretization. Acta Numerica 20 (2011) 1-119.

[8] R. Dautray and J.-L. Lions. Mathematical Analysis and Numerical Methods for Science and Technology Vol. 5. Springer-Verlag, Berlin, 1992.

[9] K. Deckelnick, G. Dziuk, and C. M. Elliott. Computation of geometric partial differential equations and mean curvature flow. Acta Numerica 14 (2005) 139-232.

[10] K. Deckelnick, A. Günther, and M. Hinze. Finite element approximation of Dirichlet boundary control for elliptic PDEs on two- and three-dimensional curved domains. SIAM J. Control Optim. 48 (2009) 2798-2819.

[11] G. Dziuk and C. M. Elliott. Finite elements on evolving surfaces. IMA J. Numer. Anal. 27 (2007) 262-292.

[12] L. C. Evans. Partial Differential Equations. Graduate Studies in Mathematics 19, AMS, 1998.

[13] C. M. Elliott and T. Ranner. Finite element analysis for a couple bulk-surface partial differential equation. IMA J. Numer. Anal. 33 (2013) 377-402.

[14] K. Fellner, W. Prager, and B. Q. Tang. The entropy method for reaction-diffusion systems without detailed balance: first order chemical reaction networks. Preprint 2015, arXiv:1504.08221.

[15] K. Fellner, S. Rosenberger, and B. Q. Tang. A reaction-diffusion system modeling asymmetric stem-cell division: existence, uniqueness, numerical simulation and rigorous quasi-steady-state approximation. Preprint 2014, arXiv:1408.1303. 
[16] E. Friedmann, R. Neumann, and R. Rannacher. Well-posedness of a linear spatio-temporal model of the JAK2/STAT5 signaling pathway. Comm. Math. Anal. 15 (2013) 76-102.

[17] R. Ghez and G. H. Gilmer. An Analysis of Combined Surface and Volume Diffusion Processes in Crystal Growth II. Asymmetric Capture at Steps. J. Cryst. Growth B 93 (1974) 93-109.

[18] A. Glitzky and K. Gärtner. Energy estimates for continuous and discretized electro-reaction-diffusion systems. Nonlin. Anal. 70 (2009), 788-805.

[19] A. Jüngel and S. Schuchnigg. Entropy-dissipating semi-discrete Runge-Kutta schemes for nonlinear diffusion equations. Preprint 2015, arXiv:1506.07040.

[20] B. Mayer, G. Emery, D. Berdnik, F. Wirtz-Peitz, and J. Knoblich. Quantitative analysis of protein dynamics during asymmetric cell division. Curr. Biology 15 (2005) 1847-54.

[21] U. Mayor, N. R. Guydosh, C. M. Johnson, J. G. Grossmann, S. Sato, G. S. Jas, S. M. Freund, D. O. Alonso, V. Daggett, and A. R. Fersht. The complete folding pathway of a protein from nanoseconds to microseconds. Nature, 421 (2003) 863-867.

[22] I. L. Novak, F. Gao, Y. S. Choi, D. Resasco, J. C. Schaff, and B. M. Slepchenko. Diffusion on a curved surface coupled to diffusion in the volume: Application to cell biology. J. Comput. Phys. 226 (2007) 1271-1290.

[23] L. R. Scott. Finite element techniques for curved boundaries. PhD. Thesis, MIT, Cambridge, MA, 1973.

[24] L. Tartar. An Introduction to Sobolev Spaces and Interpolation Spaces. Lecture Notes of the Unione Matematica Italiana, Springer, 2007.

[25] V. Thomee. Galerkin finite element methods for parabolic problems. 2nd ed. Springer Series in Computational Mathematics, Springer-Verlag, Berlin Heidelberg, 2006.

[26] M. F. Wheeler. A priori $L_{2}$ error estimates for Galerkin approximations to parabolic partial differential equations. SIAM J. Numer. Anal., 10:723-759, 1973.

[27] F. Wirtz-Peitz, T. Nashimura, and J. Knoblich. Linking cell cycle to asymmetric division: Aurora-A phosphorylates the Par complex to regulate numb localization. Cell 135 (2008) 161-173.

Herbert Egger, AG Numerical Analysis and Scientific Computing, Department of Mathematics, TU Darmstadt, Dolivostr. 15, 64293 Darmstadt, Germany.

E-mail address: egger@mathematik.tu-darmstadt.de

Klemens Fellner, Institute of Mathematics and Scientific Computing, University of Graz, HeinRichstrasse 36, 8010 Graz, Austria.

E-mail address: klemens.fellner@uni-graz.at

Jan-Frederik Pietschmann, Institute for Applied Mathematics, Department of Mathematics and Computer Science, University of Münster, Orleansring 10, 48149 Münster, Germany.

E-mail address: pietschmann@mathematik.tu-darmstadt.de

Bao Q. Tang, Institute of Mathematics and Scientific Computing, University of Graz, Heinrichstrasse 36, 8010 Graz, Austria, and Faculty of Applied Mathematics and Informatics, Hanoi University of Science and Technology, 1 Dai Co Viet, Hai Ba Trung, Hanoi, Vietnam.

E-mail address: quoc.tang@uni-graz.at 